\title{
The Trend Is Your (Imaginary) Friend - A Behavioral Perspective on Technical Analysis
}

\author{
Sebastian Ebert* $\quad$ Christian Hilpert ${ }^{\dagger}$
}

\begin{abstract}
Technical analysts, or chartists, aim at predicting future prices from past prices. To this end, for example, they draw resistance levels and Moving Average (MA) lines into stock price charts. We show that the widely employed MA cross-over rule is consistent with prospect theory preferences even when prices do not move in trends and when stock trading is unattractive to all rational expected utility maximizers. While chartists often argue that market participants being less than fully rational explains why technical analysis is profitable, this paper shows that technical analysis may be attractive - even when not profitable - to investors who are less than fully rational.

Keywords: Behavioral Finance, Decision Making under Risk, Downside Risk, Moving Average, Prospect Theory, Technical Analysis
\end{abstract}

\footnotetext{
${ }^{*}$ Tilburg University, Department of Finance, Room K 913, PO Box 90153, 5000 LE Tilburg, The Netherlands. Phone: +31(0)13 466 3126, Email: S.Ebert@uvt.nl

${ }^{\dagger}$ Institute for Financial Economics and Statistics, and Bonn Graduate School of Economics, University of Bonn, Adenauerallee 24 - 42, D-53113 Bonn, Germany; Email: chilpert@uni-bonn.de. Tel: +49 228 73 7994, Fax :+49 228 73 5048. We thank seminar participants at the University of Alabama, Georgia State University, and Bonn for helpful comments.
} 
Technical analysis has the objective to predict future prices from historical price data, trading volume, and statistics thereof. Fundamental analysis, on the other hand, is based on economic fundamentals as given by macroeconomic or balance sheet data. According to a recent survey of 678 fund managers by Menkhoff [2010), 86\% of fund managers rely on technical analysis. For $26 \%$ of surveyed fund mangers technical analysis is the most important criterion when making investment decisions. Many daily newspapers regularly present technical analysis of selected assets. Online brokers present price charts of the securities that customers select, and provide tools to visualize technical indicators. The Journal of Technical Analysis is a practitioners' journal on technical analysis since 1978 .

At the same time, the drawing of resistance and support levels as well as moving average (MA) lines into stock price charts to predict future prices appears odd to many people. Academics are generally skeptical towards technical analysis. Predicting future prices from past prices constitutes a futile attempt in light of the efficient market hypothesis even in its weak form (Fama) 1970, Jensen 1978).

Against this criticism, technical analysts bring forward that research in behavioral finance has questioned market efficiency. Markets are not fully rational, assets may be mispriced, and such mispricings may exist for a long time. Indeed, prices may differ from their fundamental value because of limits to arbitrage. Possible reasons are noise trader risk (e.g. De Long, Shleifer, Summers, and Vishny 1990) or agency problems that cause arbitrageurs to act myopically (Shleifer and Vishny 1997). Various models predict momentum in asset returns that could potentially be exploited through trend-chasing technical trading strategies 1

The actual profitability of technical analysis is heavily debated. Jegadeesh and Titman (1993, 2001) document the profitability of a variety of momentum strategies and provide some cautious support for the behavioral models that predict predictability of stock returns. Menkhoff and Taylor (2007) survey technical analysis in foreign exchange markets. They provide evidence that technical analysis can give additional information over fundamental factors, and thus be profitable $2^{2}$ The survey of Park and Irwin (2007) provides an overview of studies on the profitability of technical trading. There are a number of older studies that report positively on technical analysis, but often suffer from econometric deficiencies like data-snooping bias. Overall, empirical results are very mixed, and people tend to have strong opinions for - or against - technical analysis.

In this paper, we are agnostic about whether technical analysis is profitable or not. We will also not take a stand on whether the field of behavioral finance indeed lends support to the application of technical analysis. In this paper, we argue that the popularity of technical analysis may also be explained by its advocates being less than fully rational. Unlike previous behavioral work that investigates the predictability of stock returns that may result in technical trading being

\footnotetext{
${ }^{1}$ Momentum may arise, for example, because investors under- and overreact to news (Barberis, Shleifer, and Vishny 1998) because of cognitive biases that affect their belief formation. Grinblatt and Han (2005) argue that investors' tendency to hold on to assets that have lost in value and holding on to those that have gained in value may generate momentum in asset returns.

2 Menkhoff, Sarno, Schmeling, and Schrimpf (2012) find strong momentum in a cross-section of currency returns, and also give an extensive literature review on momentum trading.
} 
profitable, our argument is not concerned with-and independent of - profitability. In fact, even when - by assumption - technical trading is not profitable and cannot be reconciled with rational, risk averse expected utility theory preferences, it may be attractive to investors with less than fully rational preferences. This result matches the intuition of many academics who are skeptical towards technical analysis: trading based on technical indicators is consistent with its advocates being less than fully rational.

The investors we consider are less than fully rational because they have non-standard preferences as given by cumulative prospect theory (CPT, Tversky and Kahneman 1992, Kahneman and Tversky 1979). Unlike expected utility theory (EUT), CPT posits that investors do not evaluate absolute wealth levels, but gains and losses relative to a reference point. In evaluating the risky returns of an investment, the reference point is often zero return, the risk-free return, or expected return. The CPT utility function is concave for gains and convex for losses, which implies diminishing sensitivity to both gains and losses. In addition, the utility function is kinked at the reference point and steeper over losses than over gains. This implies that people are more sensitive to losses than to gains, which is referred to as loss aversion. Moreover, prospect theory investors behave as if their decisions are based on transformed probabilities. These transformed probabilities are obtained from objective probabilities by applying a so-called weighting function. The main effect of this probability weighting is that small probabilities of extreme returns are overweighted which results in a preference for lottery-like, or right-skewed, payoffs.

Why may trading based on technical indicators be attractive to prospect theory investors even when prices do not move in trends? We show that trend-chasing trading strategies shape the distribution of trading proceeds in a way which is desirable for CPT investors. This effect is so strong that even technical trading of a stock with zero excess return - which is not attractive according to any trading strategy to a risk-averse EUT investor-is attractive to CPT investors.

We will illustrate this result along the lines of the arguably most popular technical trading strategy, the moving average cross-over strategy (MA strategy, for short). However, from our analysis it will become evident that similar results also apply to other strategies that try to chase momentum. A MA line indicates how the average of a fixed number of most recent closing prices of a stock develops over time. The MA cross-over strategy is based on the crossings of two such MA lines which indicate a short-term and a long-term average, respectively. The cross-over MA strategy gives a buy signal when the short-term MA line crosses the long-term MA line from below, which is the case when recent prices have increased sharply. When subsequently the short-term MA crosses the long-term MA line from above, this corresponds to a sell signal. The MA strategy thus chases momentum as it buys recent winners and sells recent losers $3^{3}$ When an investor trades

\footnotetext{
${ }^{3}$ We want to emphasize that this paper is quite different in focus from recent prospect theory papers on the disposition effect such as Barberis and Xiong (2009, 2012) or Henderson (2012). The disposition effect refers to investors being more inclined to sell stocks that have gained in value and hold on to stocks that have lost in value. These models largely focus on the optimal selling decision, which is determined endogenously from preferences. Moreover, these models are without probability weighting. In the current paper, we show that an exogenously specified technical trading strategy is attractive to CPT investors, and this effect is largely driven by probability weighting.
} 
a stock as suggested by the MA strategy, we will also say that he "trades the stock MA."

The result that technical trading is consistent with $\mathrm{CPT}$ even when it cannot be reconciled under rational preferences is made in two steps. The first step consists of obtaining the return distribution from trading according to the MA for various financial market models as well as for actual data. The second step consists of verifying that this distribution is indeed attractive to prospect theory investors.

In the first step, for comparative reasons, we benchmark the MA strategy to a totally random trading strategy. This benchmark strategy is a random buy-and-hold strategy whose buying and sell times are identical in distribution to that generated by the MA strategy. Therefore, also the holding time is identical in distribution to that of MA. However, unlike is the case for the MA strategy, for the benchmark strategy buying and sell time are independent of the stock price evolution. This allows us to study in isolation the effect of timing as suggested by MA on the return distribution.

The main result we obtain in step 1 is that trading MA skews the return distribution strongly to the right. Skewness in asset returns implies a large probability of small negative returns and a small probability of very high returns. For stock price dynamics as in the Black-Scholes model, for example, returns are log-normally distributed and thus right-skewed. Not surprisingly, also the distribution of our benchmark buy-and-hold strategy is right-skewed. Trading the stock MA, however, skews the stock's distribution much further to the right.

This result holds accordingly for short-selling. For example, short-selling a log-normal stock buy-and-hold results in a left-skewed return distribution. Shorting the stock based on MA buy and sell signals skews the distribution of trading proceeds to the right such that this distribution in fact becomes right-skewed. The result that trading MA strongly skews the return distribution to the right is robust towards different underlying stock price dynamics with and without trend or autocorrelation, different time horizons, and is also found in real data on the STOXX Europe 600 stocks.

In the paper, we also explain why trading MA skews the return distribution to the right, and illustrate why the effect is not specific to the dynamics of the stock price process. Trading MA features a subtle asymmetry in the way stocks are sold subsequent to a buy signal. A stock whose price rises is not sold until until it experiences a significant loss. Thus the right tail of the return distribution is pronounced. After a sudden price decline, the stock is sold immediately. This limits the downside of the investment by shortening and thickening the left tail.

The second step of our analysis consists of verifying that the MA return distribution is attractive to CPT investors, even when it is not attractive to rational investors. To this end, we employ the numerical methodology developed in Barberis (2012), which also allows us to determine the drivers of this result. Recent theoretical papers have argued that prospect theory implies a strong preference for skewed payoffs; e.g. Barberis and Huang (2008), Ebert and Strack (2012), or Barberis (2013). There is a growing amount of evidence on skewness preference in financial decision situations, ranging from CEO's project choices (Schneider and Spalt 2012) to initial public offerings (Green and Hwang 2012). In this spirit, our results show that the popularity of technical trading strategies 
like MA may be explained by strong skewness preference. Schneider and Spalt (2012) also use the term "longshot bias" to refer to CEO's excessive skewness preference.

On a more general level, this paper offers a new explanation for why technical analysis and trend-chasing are popular; being that its advocates and applicants have less than fully rational preferences. This argument is very different from a well-known behavioral argument on technical analysis, one which is often made by its advocates; that technical analysis may be valuable because markets are not efficient, potentially due to other market participants being less than fully rational. Having this in mind, we want to emphasize that both arguments are by no means mutually exclusive. As we illustrate through various robustness checks, the effect that trading MA shapes the return distribution in a way desirable to prospect theory investors holds irrespective of whether the assumptions for why MA is applied are met. If MA is highly profitable because prices do move in trends, then the rational reason to trade MA (MA being highly profitable) is reinforced by the less than fully rational we point out (prospect theory preferences). For the main part of the paper we wish to disentangle these effects and study the less than fully rational reason we point out in isolation, and show that it is sufficient to make MA attractive to CPT investors when rational EUT traders do not trade the stock at all. Also other, non-behavioral arguments in favor of technical analysis may be valid. Our conclusion is that boundedly rational investor preferences are one potential - however sufficient - reason for the popularity of technical analysis. This reason may or may not be accompanied other, rational reasons.

The paper proceeds as follows. Section I defines the MA strategy within a simple financial market model as well as investor preferences. In Section II] we show, and explain why, trading MA skews the distribution of trading proceeds to the right. In Section III we show that trading MA is attractive to prospect theory investors even when prices do not move in trends and when MA is not profitable. In Section [V] we show that an MA short-sale strategy likewise skews to the right and is attractive to prospect theory investors. Section $\mathrm{V}$ then shows that these results are robust towards different specifications of the time horizon, variations of the MA strategy, and alternative stock price dynamics. In particular, our results stay qualitatively the same when prices do move in trends, and they are also found in real data on STOXX Europe 600 stocks. Section VI concludes.

\section{Technical Trading Strategies and Investor Preferences}

We first define the double-crossover MA strategy in Subsection I.A and illustrate it by means of an example. Subsection I.B defines the investor's preferences. In Subsection I.C, we discuss MA in a simple, geometric Brownian Motion model that will be analyzed in the main part of this paper. 


\section{A. The double-crossover MA trading strategy}

Let $S_{t}$ denote the time $t$ price of a stock $S$. The value of the MA line of stock $S$ at a given time $t$ with length $n$ is the average closing price of the past $n$ closing prices:

$$
M A(t, n)=\frac{1}{n} \sum_{i=1}^{n} S_{t-i} .
$$

As time moves on, the observations used to calculate the average are replaced with newer observations of the stock price. The MA (line) of a fixed length $n$ refers to the function $t \mapsto M A(t, n)$ and indicates how the recent average stock price evolves over time. Short-term MAs ( $n$ small) are more responsive to recent price changes than long-term MAs ( $n$ large), because more recent observations have higher weight in the computation of the average in equation (1).

MAs smooth out short-term fluctuations in the stock price and thus indicate, depending on their length, short-term or long-term trends in the stock price. The perhaps best-known trading strategy based on MAs is the double-crossover MA strategy. In this paper, we refer to it as "MA strategy" for short. The MA double-cross-over strategy is based on two MAs with different time spans $n_{\text {long }}>n_{\text {short }}$. A buy signal occurs when the short-term MA crosses the long-term MA from below, which indicates a short-term upward trend (relative to the long-term trend). Similarly, a sell signal occurs when the short-term MA crosses the long-term MA from above, indicating that the short-term upward trend is over. Formally:

Definition 1 (MA Strategy): The MA strategy indicates a buy signal at time $t$ if the short-term MA line crosses the long-term MA line from below:

$$
M A\left(t-1, n_{\text {short }}\right)<M A\left(t-1, n_{\text {long }}\right) \text { and } M A\left(t, n_{\text {short }}\right) \geq M A\left(t, n_{\text {long }}\right)
$$

The MA strategy indicates a sell signal at time $t$ if the short-term MA line crosses the long-term MA line from above, that is,

$$
M A\left(t-1, n_{\text {short }}\right)>M A\left(t-1, n_{\text {long }}\right) \text { and } M A\left(t, n_{\text {short }}\right) \leq M A\left(t, n_{\text {long }}\right) .
$$

If we set the time a buy-signal occurs to $t=0$, the holding time $\tau_{M A}$ denotes the first time after $t=0$ when a sell-signal occurs.

A popular parametrization is $n_{\text {short }}=50$ for the length of the short-term MA and $n_{\text {long }}=200$ for length of the long-term MA 4 As MA lines are exclusively based on time series data, they cannot indicate trends that result from cross-sectional phenomena like a negative return correlation for winners and losers. They may indicate upward trends that are due to autocorrelation, or trends that are simply the result of the stock having a high unconditional mean.

\footnotetext{
${ }^{4}$ While 200 is clearly the standard for the long-term MA, technicians sometimes use smaller values like 22 or 37 for $n_{\text {short }}$. Using shorter values than 50 for $n_{\text {short }}$ does not change the principal argument we make in this paper, but rather amplifies our results.
} 
Figure 1 illustrates the MA strategy along the lines of the Walmart common stock (ISIN: US9311421039) trajectory of daily closing prices on NYSE for 2008-2013. The figure also shows the prices for which the MA strategy indicates buy and sell signals. A profitable trade according to the MA strategy would have been buying the stock at the buy signal after July 2011 for $\$ 58$ and selling it for $\$ 69$ in March 2013. A losing trade would have resulted from buying for $\$ 54$ in late 2010 and selling for $\$ 53.40$ in early summer of 2011 . Minor gains would have resulted from buying in October 2009 for $\$ 51$ and selling for $\$ 53$ in late 2010.

Figure 1: MA buy and sell signal for Walmart from January 2008 to April 2013. This graph shows the 50-day MA (dashed line) and 200-day MA (solid line) lines computed from the Walmart stock's daily closing prices (solid line) on NYSE from January 2008 to April 2013. The graph also shows the buy and sell prices of the MA strategy.

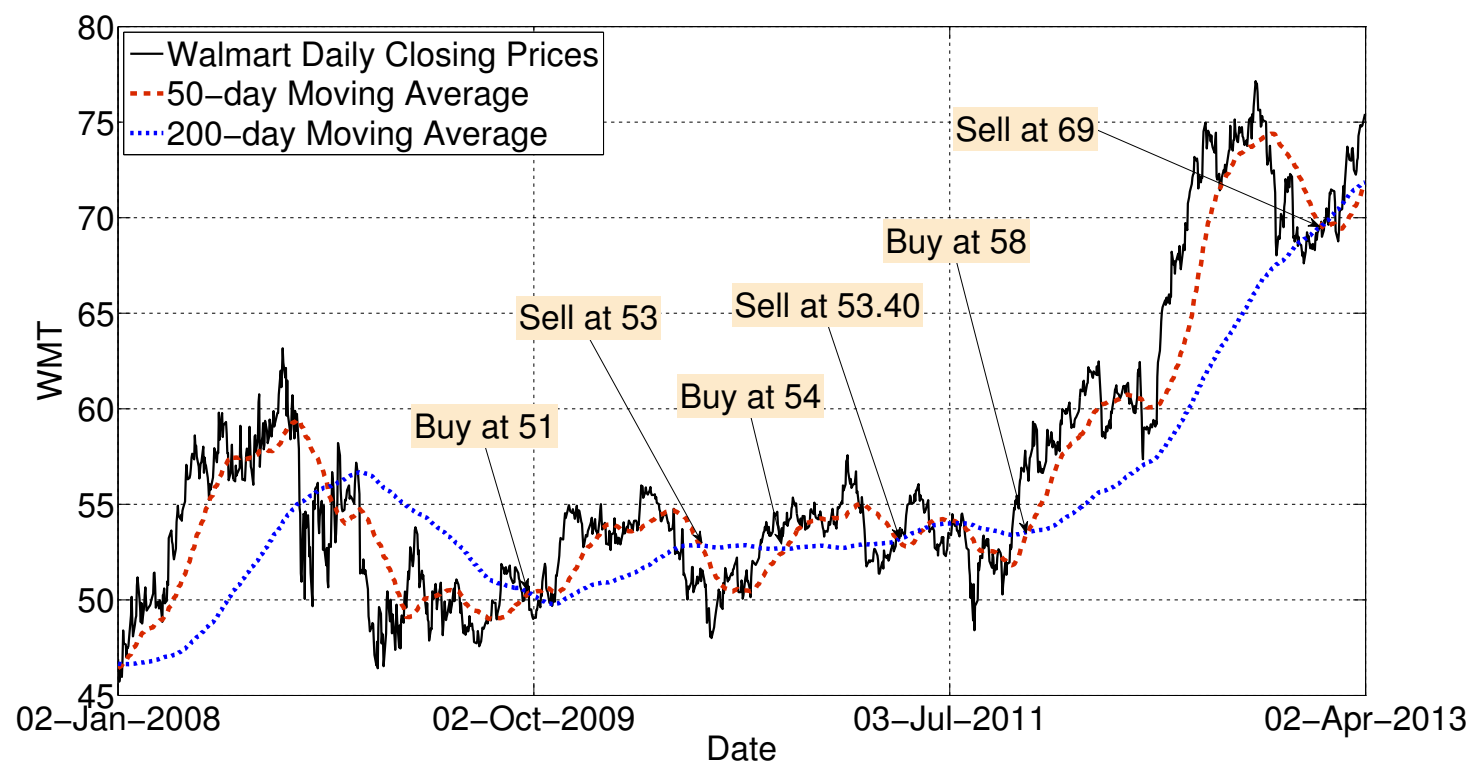

\section{B. Investor Preferences}

We consider an investor who thinks of his investment history in terms of different investing episodes. Each episode is defined by the name of the investment, the purchase price, and the sale price; cf. Barberis and Xiong (2012). Rather than taking a portfolio view, the investor evaluates the investment in isolation. This view is consistent with the ideas of narrow framing and mental accounting. Shefrin and Statman (1985), for example, argue that when an investor sells a stock, he closes a mental account that was opened when he first bought the stock. The moment of sale is therefore a natural time at which to evaluate the investment episode. Episodes that result in gains bring positive utility, while losses bring negative utility. In this paper, we study how an investor evaluates an episode when buying and selling according to the MA strategy. In particular, the investor has CPT preferences over returns from trading MA.

The CPT investor evaluates the absolute returns on his investment relative to a reference return $r$. Reasonable choices for $r$ are zero return, risk-free return, or expected return. We will generally 
assume that the investor views positive absolute returns as gains and negative absolute returns as losses. This implies that the reference return is zero, i.e., any trade at which the stock is sold for more than the purchase price brings positive utility. In the robustness section, we also discuss other reference points.

Consider a prospect (i.e., a risky return) $X$ with ordered outcomes $x_{1}>x_{2}>\ldots x_{k} \geq r>$ $x_{k}>\ldots>x_{n}$ and respective probabilities $p_{1}, \ldots, p_{n}$. Returns $x_{k+1}, \ldots, x_{n}$ are larger than the reference return $r$ and thus referred to as gains. Outcomes $x_{k}, \ldots, x_{n}$ are referred to as losses. The CPT-utility is given by

$$
C P T(X)=\sum_{i=1}^{n} \pi_{i} u\left(x_{i}-r\right)
$$

with decision weights $\pi_{i}$ and a utility function $u$ defined as follows. The value function $u$ is given by

$$
u(x-r)= \begin{cases}(x-r)^{\alpha} & \text { if } x \geq r \\ -\lambda(-(x-r))^{\alpha} & \text { if } x<r\end{cases}
$$

with "loss aversion parameter" $\lambda \geq 1$ and utility curvature parameter $\alpha \in(0,1] . \alpha \in(0,1]$ implies that the utility function is concave over the region of gains and convex over the region of losses. This implies diminishing sensitivity towards both gains and losses. $\lambda \geq 1$ implies that the utility function is steeper in the region of losses than in the region of gains, which implies that losses loom larger than gains. The left panel of Figure 2 shows the value function for pronounced loss aversion $(\lambda=2.5)$ and pronounced curvature $\alpha=0.5$. Estimates in the literature vary significantly both within studies and within subjects, but benchmark values often reported are the median values of the subjects in the Tversky and Kahneman (1992) study: $\lambda=2.25$ and $\alpha=0.88$.

Finally, CPT does not process objective probabilities linearly as does EUT, but replaces the objective probabilities $p_{i}$ with decision weights $\pi_{i}$. The $\pi_{i}$ in equation (4) are given as 5

$$
\begin{array}{ll}
\pi_{i}=w\left(p_{1}+\ldots+p_{i}\right)-w\left(p_{1}+\ldots+p_{i-1}\right), & i=1, \ldots, k \\
\pi_{i}=w\left(p_{i}+\ldots+p_{n}\right)-w\left(p_{i+1}+\ldots+p_{n}\right), & i=k+1, \ldots, n
\end{array}
$$

where $w$ is called (probability) weighting function. Tversky and Kahneman (1992) propose the following weighting function:

$$
w(p)=\frac{p^{\delta}}{\left(p^{\delta}+(1-p)^{\delta}\right)^{\frac{1}{\delta}}}
$$

with a probability distortion parameter $\delta \in(0,1]$. The right panel of Figure 2 shows plots of the weighting function for different values of $\delta$. For $\delta=1$ (solid line) the weighting function is

\footnotetext{
${ }^{5}$ In the computations of $\pi_{1}$ and $\pi_{n}$, let $w\left(p_{1}+\ldots+p_{0}\right)=w\left(p_{n+1}+\ldots+p_{n}\right) \equiv 0$.
} 
linear which implies $\pi_{i}=p_{i}$ for all $i=1, \ldots, n$, i.e., no probability distortion. For $\delta<1$ the function is inverse-S-shaped. This results in decision weights that overweight the probabilities of small and extreme outcomes, i.e., the tails of the evaluated distribution are overweighted. Tversky and Kahneman (1992) estimated a value of $\delta=0.65$ for their median subject which implies a pronounced inverse-S-shape for the weighting function $w$.

Figure 2: CPT value and weighting functions. The left hand graph shows the CPT-value function for a loss aversion parameter $\lambda=2.5$ and a S-shape parameter of $\alpha=0.5$. The right hand graph shows the CPT-probability distortion function for the case without probability distortion (solid line), an intermediate probability weighting with $\delta=0.65$ (dashed line), and a strong probability weighting of $\delta=0.4$ (dotted line).
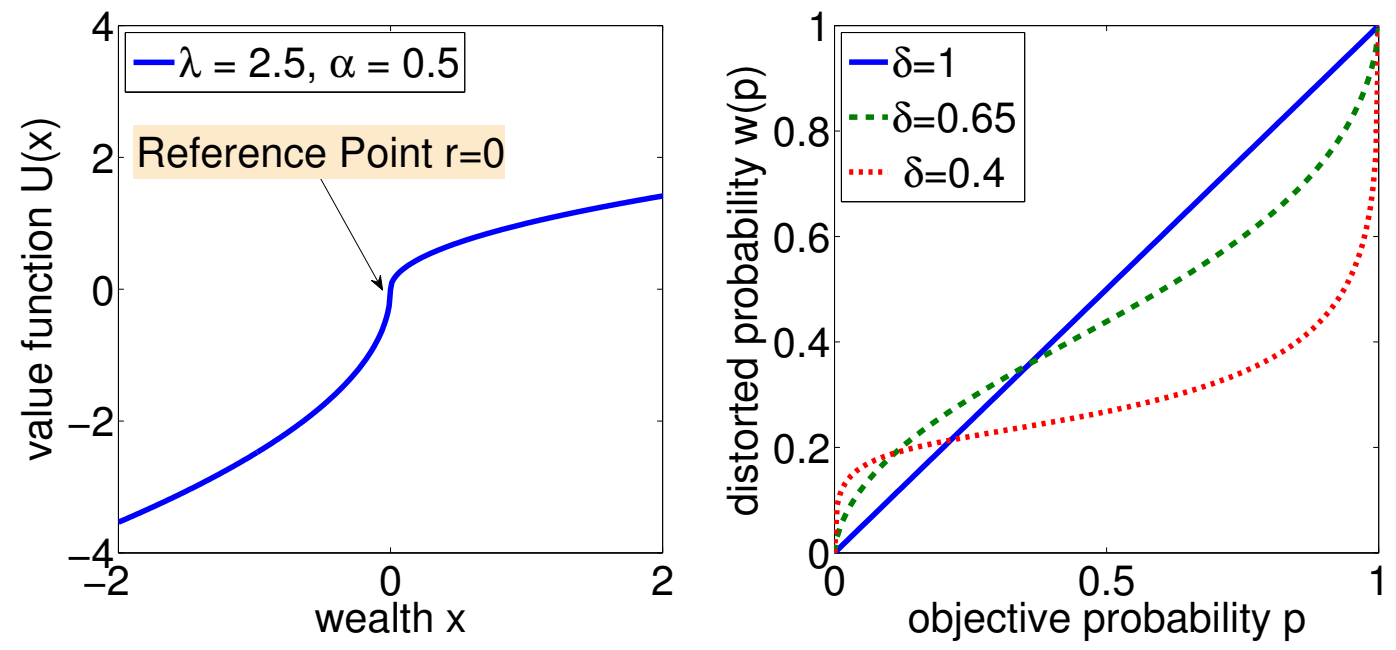

\section{MAs in a Simple Financial Market Model}

Suppose the stock price follows a geometric Brownian motion, i.e.,

$$
d S_{t}=\mu S_{t} d t+\sigma S_{t} d W_{t}, \quad \text { with } S_{0}>0
$$

where $W$ is a standard Brownian motion, $S_{0}$ is the initial stock value, and $\mu$ and $\sigma$ represent the drift and volatility of the stock, respectively. In the simulations we discuss in the main part of the paper, we consider an unattractive stock without drift, that is, $\mu=0$. If the investor does not invest in the stock, he earns a risk-free return of zero. In this setup, which is clearly artificial, the stock earns zero excess return. While our results also hold for other, more realistic specifications, they are best illustrated in this simple setup. We will illustrate the robustness of our results towards more realistic setups in Section $\mathrm{V}^{6}$

Why do we choose to illustrate our results in this abstract setup? The geometric Brownian motion is Markovian and therefore, by assumption, the stock price does not move in trends. This

\footnotetext{
${ }^{6}$ Similarly, Barberis (2012) considers a casino which offers fair gambles. Also this stylized assumption does not affect any of his results, but rather allows for clear-cut interpretations and for identifying the drivers of the model's results.
} 
renders the original purpose of trend-chasing trading strategies like MA moot. When, in addition, a stock offers zero excess return, the holding time of the stock does not affect expected returns. Therefore, by Doob's optional sampling theorem it follows that every trading strategy (and MA in particular) results in an expected return of zero. Therefore, no rational risk-averse expected utility maximizer would invest in the stock. However, and this is what we show in this paper, trading MA will still be beneficial for investors with less than fully rational (CPT) preferences. In other words, we show that CPT can explain the popularity of technical trading even when, by assumption, the fundamental reasons for it are not met.

When in Section $\mathrm{V}$ we relax or vary some of these assumptions, results change in the expected directions. For example, when the stock offers excess return, then trading MA will be even more attractive to CPT investors, and will also be attractive to rational investors who are not too riskaverse. If we assume that prices are autocorrelated, then trading MA becomes profitable, and more attractive to CPT and EUT investors alike. Disentangling the drivers that make stock investment attractive in these settings is, however, difficult, which is why for our main illustrations we prefer the simple and abstract setup outlined above.

For comparative reasons, we also analyze the investor's utility from trading stocks randomly according to a buy-and-hold strategy, also referred to as benchmark strategy. The buy and sell times of this benchmark strategy are uncertain and identical in distribution to those generated by the MA strategy 7 Therefore, also the holding time of the buy-and-hold strategy is identical in distribution to that of MA. The only difference is that the benchmark strategy buy and sell times are independent of the stock price evolution. Consideration of the benchmark strategy allows us to study in isolation the effect of timing as suggested by MA on the return distribution 8

\section{Trading MA When Prices do not Move in Trends}

In this section and the next, we show that trading a stock MA is consistent with CPT preferences even when prices do not move in trends and when rational, risk-averse EUT investors do not invest in the stock. To this end, we first compute the distribution of trading proceeds that results from trading MA. Then, in the next section, we analyze the utility of this distribution to EUT and CPT investors.

Note that, even for simple asset dynamics like the geometric Brownian motion, the return distribution from trading MA is not available in closed form. The MA is constructed as the average of lognormally distributed random variables, whose distribution is not known. This makes recovering the distribution implied by the MA strategy, which is based on the crossings of two such MAs, difficult 9 As regards the CPT analysis of trading MA we will pursue on the next section, the

\footnotetext{
${ }^{7}$ In the Markovian setting specified in this section, the buy time is immaterial. For some of the robustness checks we conduct in Section $\mathrm{V}$ however, the buy time matters.

${ }^{8}$ In Section V w w study MA in a finite time horizon framework, and also compare to a buy-and-hold strategy subject to a random, exponentially distributed liquidity shock. This does not affect our results quantitatively.

${ }^{9}$ In specific settings, however, it is possible to obtain analytical results. Zhu and Zhou (2009) compute the expected log-utility for a moving average strategy based on just one, geometric MA. For general power utility they
} 
nonlinear probability weighting embedded in CPT poses a further obstacle to analytical treatment. In fact, it is fairly obvious that under probability distortion an analysis of the MA trading strategy or even one of its simpler variants will not be analytically tractable.

We thus analyze our model (and also the robustness checks discussed in Section V) numerically for a Monte Carlo sample of size $M=100,000$. In the main part of this, paper we assume that the stock follows a zero-drift geometric Brownian motion with volatility $\sigma=30 \%$. The short-term MA has a length of 50 days and the long-term MA has a length of 200 days. To obtain the MA return distribution we thus simulate prices for at least 200 days so that the short-term and longterm MAs are defined. We then continue simulating until the MA strategy gives a buy signal and, subsequently, a sell signal. This way we also obtain the distribution of the waiting time until the MA strategy gives a buy signal (referred to as the buy time distribution) as well as the distribution of the holding time (sell time minus buy time).

Figure 3 shows the distribution of trading proceeds for the MA strategy (solid line) and the benchmark strategy (dashed line). Since the stock has zero drift and does not move in trends, by

Figure 3: Return Distribution of MA strategy. This graph shows the return distribution for the MA(50,200) strategy (solid line) and the benchmark strategy (dashed line). The stock follows a geometric Brownian motion with drift $\mu=0$ and a volatility of $\sigma=0.3$. The expected return of both strategies is zero, and the average holding time is 148 days. The skewness of the $\operatorname{MA}(50,200)$ strategy is 5.52 and the skewness of the benchmark strategy is 1.33 .

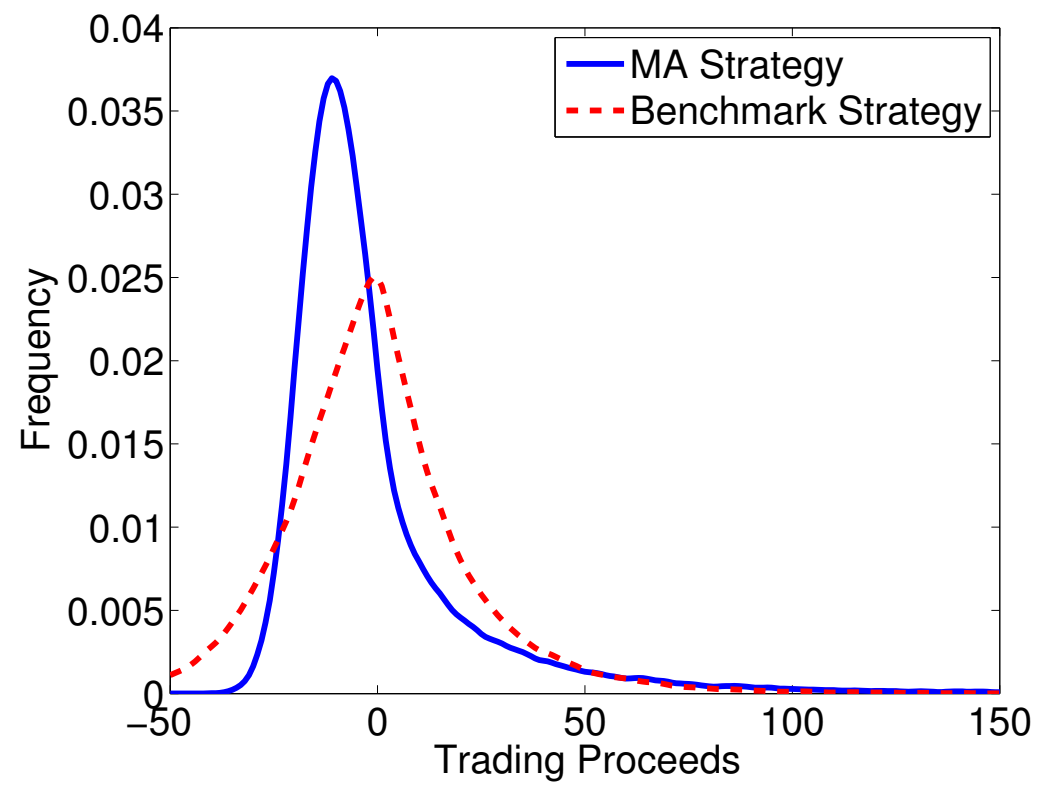

Doob's optional sampling theorem the expected return of both the MA and the benchmark strategy are zero.

While trading MA cannot generate excess return in this setting, it does have an effect on the shape of the return distribution. The overall effect of trading MA is that, as compared to random trading as is in the case of the benchmark strategy, the distribution of trading proceeds obtain approximately analytical results. 
is strongly skewed to the right. Trading MA reduces the probability of returns that are less than $-35 \%$ to almost zero $(0.029 \%)$. At the same time, the probability of moderately negative returns is substantial: While the left tail of the distribution is short, it is also very thick. The right tail of the distribution, on the other hand, is long but very lean. The far right tail lies slightly above that of the benchmark strategy, while the probability of moderate gains is smaller. The third standardized central moments compute to 5.52 and 1.33 for the benchmark and the MA strategy, respectively.

Figure 4: Sales timing implied by trading MA. The top row shows stylized stock price trajectories (solid line) and corresponding evolutions of 50-day (dotted line) and 200-day (dashed line) MA lines. The bottom row highlights in gray different parts of the $M A(50,200)$ return distribution shown in Figure 3 . The shape of the return distribution in the highlighted parts can be related to the time of sale that the MA strategy dictates for stock price evolutions as in the above panel.

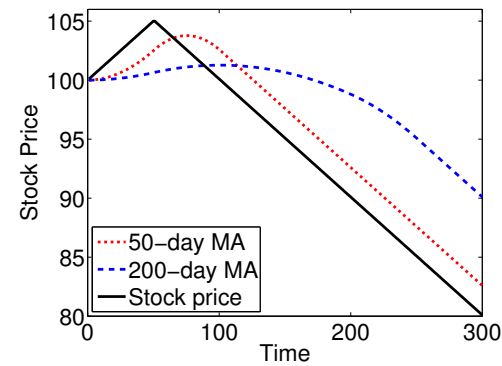

(a) Heavy Losses

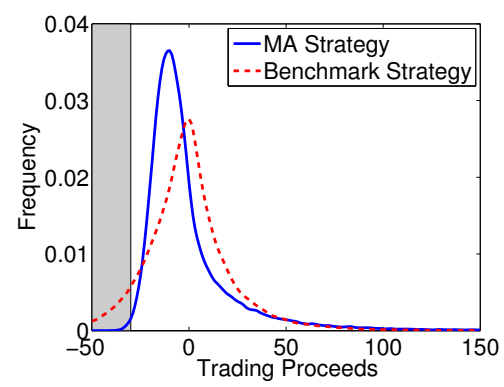

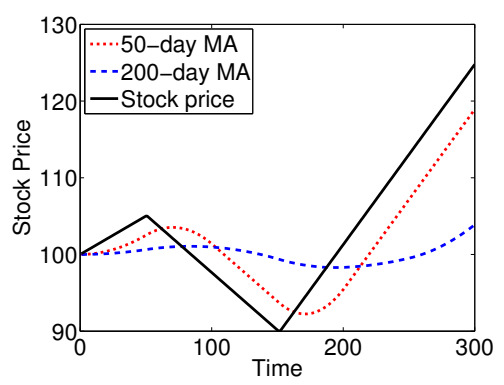

(b) Small Gains/Losses

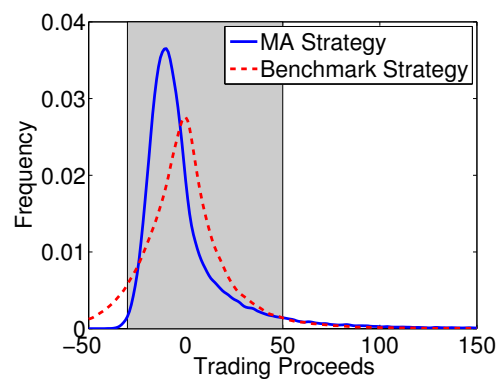

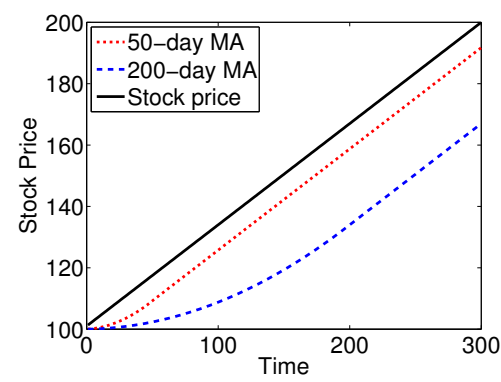

(c) Large Gains

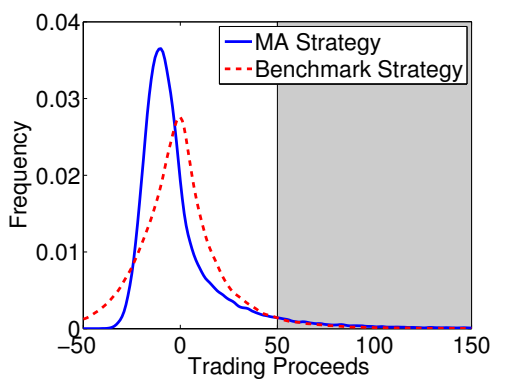

Why does trading MA skew the distribution of trading proceeds to the right? What is the intuition behind the asymmetric effect that trading MA has on the tails of the return distribution? Figure 4 provides the intuition for this result by considering three stylized stock price trajectories subsequent to a buy signal (shown in the upper panels of Figures $4 \mathrm{a}, 4 \mathrm{~b}$, and $4 \mathrm{c}$ ) 10 For each of the three scenarios, the lower panel shows again the MA return distribution from Figure 3. Each scenario explains why the MA return distribution differs in the highlighted, gray area from that of random trading as given by the benchmark strategy.

In the scenario depicted in Figure 4a, the stock price increases at first and then declines substantially. The 50-day MA line picks up the initial upward trend and rises quickly, while the increase of the 200-day MA is less pronounced. Likewise, once the stock price starts to decline, the 50-day MA adjusts quickly and turns downward. The 200-day MA is yet increasing when the price decline

\footnotetext{
${ }^{10}$ On the 199 trading days prior to the buy signal, for each of the three scenarios, the stock traded constantly at 100 while on day 200 before the buy signal it traded at 50 . Note that this indeed results in a buy signal at $t=0$. The holding time of the buy-and-hold strategy is 300 days.
} 
starts; only slowly is the 200-day MA turning downward. The sell signal occurs when the 50-day MA crosses the 200-day MA at a value above 100. At that time, the stock price has decreased to about 98 . Therefore, trading MA yields a mild negative return of $-2 \%$ in this scenario. The benchmark strategy would have resulted in a substantially negative return of $-20 \%$. This example illustrates that the MA strategy sells a stock after an episode of sufficiently rapid price decline. This explains why there is almost no probability mass on substantial losses (the highlighted area in the lower panel of Figure 4a). Only when prices decline steadily for a long time, at a rate small enough to avoid a sell-signal, MA may result in large losses.

The scenario in Figure 4b starts out just like that in Figure $4 \mathrm{a}$, but after the decline in prices from day 51 to 150 the stock recovers. As the stock has been sold by then, the investor misses upon a quite large return of $25 \%$. Instead, he realizes a moderate loss just as in the previous scenario. This explains why trading MA shifts probability mass from moderate gains to moderate losses. Again, whenever the stock price decreases sufficiently within a short period of time the stock is sold. Thus all scenarios with such an early price decline result in a mildly negative return. This effect takes probability mass from both severe losses (scenarios as depicted in the upper panel of Figure 4a and moderate gains (scenarios as depicted in the upper panel of Figure 4b to mild losses (the lower panel of Figure 4b). This explains the thick but short left tail of the MA return distribution. In fact, the probability of earning a return between $-30 \%$ and $0 \%$ in Figure 3 equals $69.25 \%$.

Finally, Figure $4 \mathrm{c}$ shows that for continuously increasing stock prices without large setbacks, the MA lines do not cross. The sluggish 200-day MA just never catches up with the 50-day MA line. Therefore, the fact that the MA strategy buys into the stock when the short-term MA lies above the long-term MA and that the short-term MA is more flexible in adjusting to recent stock price movements implies a systematic asymmetry in the way stocks are sold. Stocks that keep rising and rising are never sold, which implies a pronounced extreme upside. Losing stocks are sold quickly, which implies a restricted downside. Effectively, trading MA thus results in skewing the distribution of the overall trading experience to the right. This asymmetry in when stocks are sold has nothing to do with chasing a trend that may potentially be predicted from past prices or the MA buy signal in particular. The sell signal is based on future prices - trend-following or not. Therefore, while some investors might argue that they trade MA because they believe in prices

moving in trends, it may also be the case that they appreciate the right-skewed trading experience MA subtly imposes.

\section{Cumulative Prospect Theory Utility of MA Trading}

In this section, we verify that trading MA can be reconciled with CPT preferences. To this end, we evaluate the return distribution that results from trading MA with CPT as defined in Section I. As explained before, no risk-averse expected utility maximizer will trade stocks in this setting. Because of the non-linear probability weighting embedded in prospect theory, for CPT we 
have to evaluate the MA return distribution numerically. Our methodology for this part of the analysis is similar to that in Barberis (2012).

We restrict our attention to parameter values $(\alpha, \delta, \lambda)$ for which $\alpha \in(0,1], \delta \in[0.3,1]$, and $\lambda \in[1,4]{ }^{11}$ This parameter range covers well the parameter values that are typically estimated in empirical studies or experiments. The median estimate of Tversky and Kahneman (1992) is given by $(\alpha, \delta, \lambda)=(0.88,0.65,2.25)$. For each parameter, we discretize the parameter range specified above into 20 different, equally distant values. We then compute the CPT utility of the return distributions implied by MA and buy-and-hold for $20^{3}=8000$ different values. The CPT utility of not investing is zero in our setting.

The results of these computations are visualized in Figure 5. Figure 5a only compares the

Figure 5: Utility of Trading MA. This graph illustrates the parameter values for which a CPT investor finds trading MA attractive. For comparative reasons, part (a) indicates with a " + " the 915 out of the 8000 considered parameter values for which trading the stock randomly according to the benchmark strategy is preferred over no investment. Part (b) compares preference between a stock investment according to the benchmark strategy, the MA strategy, and no stock investment. In that case, the benchmark strategy is optimal for only 15 parameter combinations (indicated by a " + ") while the $\operatorname{MA}(50,200)$ strategy is preferred for 2541 parameter combinations (indicated by a "*").

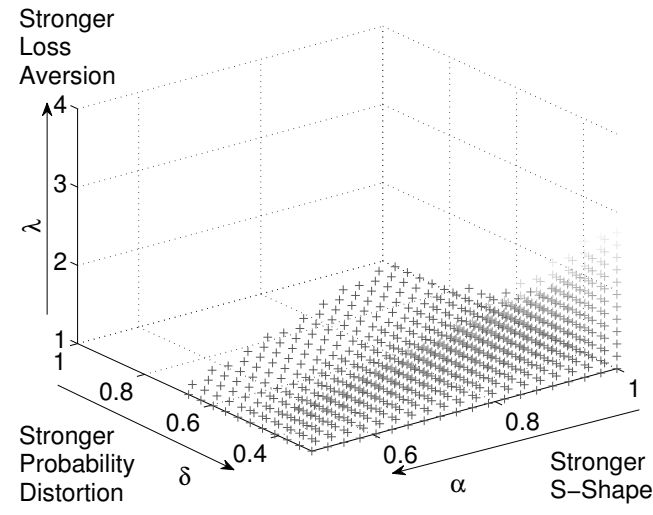

(a) Buy-and-hold vs. no investment

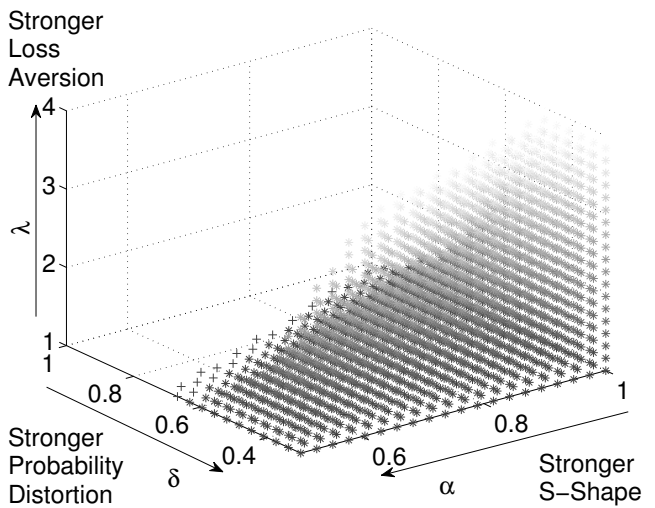

(b) MA vs. buy-and-hold vs. no investment

benchmark buy-and-hold strategy with the possibility of no investment. A "+" sign marks the parameter triples $(\alpha, \delta, \lambda)$ for which the benchmark strategy yields a higher CPT utility than no investment, i.e., positive CPT utility.

Figure $5 \mathrm{a}$ shows that trading the zero return stock buy-and-hold is attractive to only few CPT investors (for 915 out of the 8000 parameter triples). These investors' preferences are characterized by a mildly S-shaped utility function (large $\alpha$ ), pronounced probability distortion ( $\operatorname{small} \delta$ ), and mild loss aversion (small $\lambda$ ). The intuition is straightforward. Stock investment according to the benchmark strategy is risky and may result in losses bounded by the initial investment, but also in unbounded gains. A mild S-shape of the utility function implies that the marginal utility of gains decreases less rapidly. Probability weighting implies that the unbounded right tail of the distribution is significantly overweighted. And low loss aversion ensures that the investor is not too afraid of the potential losses of a risky investment.

\footnotetext{
${ }^{11} \delta \geq 0.3$ is imposed to ensure that the weighting function is increasing as first noted by Rieger and Wang (2006).
} 
Figure 5b illustrates the investors' decision when his investment opportunity set is enriched by the MA strategy. In Figure 5b, a "+" marks the parameter triples for which buy-and-hold yields the highest CPT utility. A “*” symbol marks the parameter triples for which the CPT utility of trading MA is highest. If the space for a parameter triple is left blank, the investor decides against investment because the CPT utility of buying the stock is negative for both trading it MA or buy-and-hold.

From Figure $5 \mathrm{~b}$ we see that, firstly, investing in the stock is attractive for much more CPT preference parameter triples (2556 of 8000). Secondly, almost all (2541) of these triples are "** triples. This means that those investors who prefer to invest in the stock prefer trading it MA to buy-and-hold. Only 15 out of the 2556 stock investors prefer to stay with trading it buy-and-hold when also MA is available. The key to understanding this result is to recall what trading MA does to the distribution of trading proceeds (Figure 3).

Both MA and buy-and-hold result in a right-skewed distribution of trading proceeds. This is why both strategies are generally favored by investors with a mildly S-shaped utility function, mild loss aversion, and pronounced probability weighting. Trading MA, however, results in a much more right-skewed distribution than trading buy-and-hold. This increased skewness makes MA attractive to investors with pronounced probability weighting even when they are quite loss averse or when they have a pronounced diminishing sensitivity towards large gains. Probability weighting is in fact necessary for skewness preference. Indeed, loss-averse-investors with very little probability weighting may even prefer left-skewed return distributions; this will be explained in the next section. The fact that CPT investors with mild probability weighting prefer less skewness is also the reason why for 15 investors we find that the mildly skewed buy-and-hold is more attractive than MA. Note that these 15 parameter triples indeed feature moderate levels of probability weighting.

\section{Short Selling}

In this section, we repeat the analysis of the previous two sections for the short-sale MA strategy. This is interesting because shorting a stock results in a left-skewed return distribution 12 This is in part due to the stock price dynamics we assume, but also lies in the nature of the short-sale. The upside return of a short position is limited to $100 \%$ (in case the stock becomes worthless), while the downside is unbounded (because the potential stock price increase is unbounded). This is just the opposite to the long position where the downside return is limited to $-100 \%$ and the upside is unbounded. The analysis of the short-sale MA strategy thus also sheds light on whether our observations depend upon the fact that we started out with a right-skewed return distribution as generated by geometric Brownian motion. A possible hypothesis could be that trading MA skews left-skewed distributions more to the left and right-skewed distributions more to the right; or that it has no systematic effect; or that trading MA skews returns to the right no matter what.

\footnotetext{
${ }^{12}$ The short-sale benchmark strategy is analogous to the benchmark strategy for the long position case. It buys the stock at a random time which is identical in distribution to the buy time of short-sale MA, and holds it for a time period which is identical in distribution to that of short-sale MA.
} 
The MA short-sale strategy tries to exploit negative momentum in the stock by shorting it when the 50-day MA crosses the 200-day MA from above, thereby capturing a downward trend.

Definition 2 (Short-Selling-MA Strategy): The short sale-MA strategy opens a short position in the stock when the MA lines indicate a sell signal, and closes the short position when the MA lines indicates a buy signal.

Figure 6 shows the short MA return distribution (solid line) and that of the benchmark short strategy (dashed line). The graph shows that also the short-sale MA skews the distribution of

Figure 6: Return distribution of short-sale MA. This graph shows the distribution of trading proceeds that results from short-sale MA (solid line) and the benchmark short-sale strategy (dashed line). The skewness of the short-selling-MA strategy is 1.02 and the skewness of the benchmark strategy is -1.63. The average holding time is 180 days.

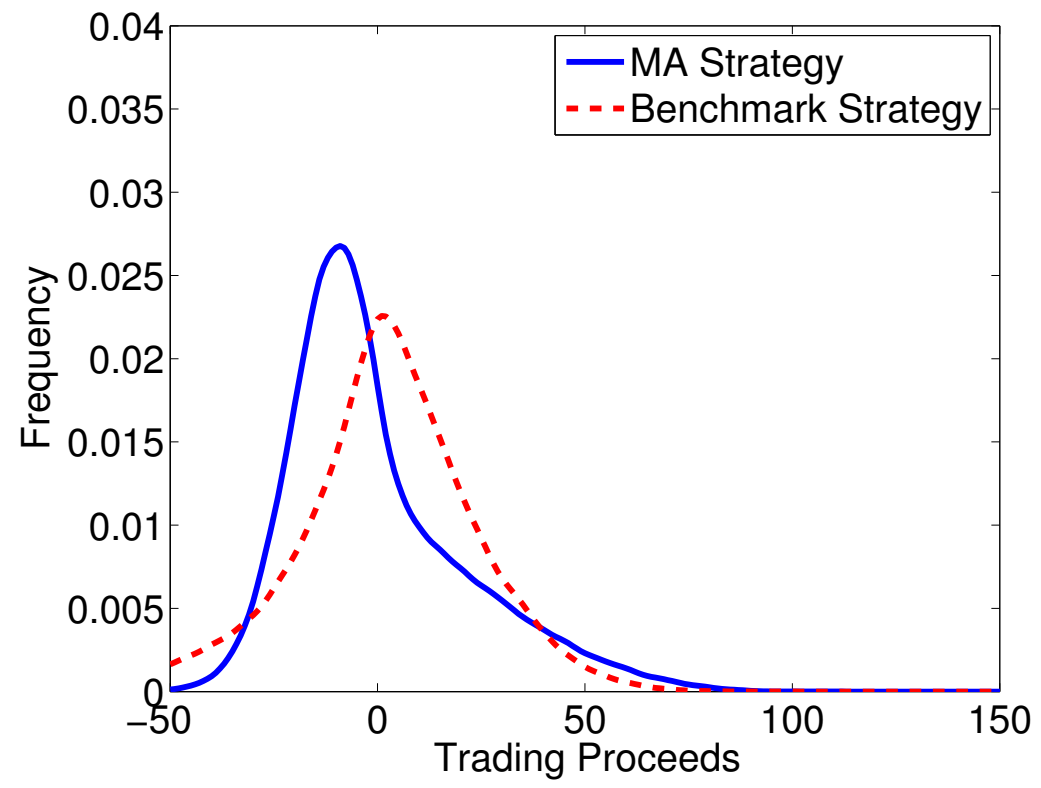

trading proceeds to the right, even though the benchmark buy-and-hold strategy is indeed leftskewed. The third standardized central moments are given by -1.63 for the buy-and-hold and by +1.02 for the MA. This result is in fact not surprising. The same logic explained along the lines of Figure 4 also applies in the case of short-sale. MA implies that the position in the stock-now a short position - is closed quickly once its value deteriorates sufficiently strongly and quickly. When the value of the position continuously rises (in case of the short sale, prices decrease steadily), the MA strategy does not yield a sell signal. Thus the upside implied by the MA strategy is more pronounced than that of the benchmark strategy. Therefore, also short-sale MA results in a rightskewed return distribution. As, due to the nature of the short-sale, the upside is less pronounced than in the case of a long position, the short-sale MA strategy results in a comparatively less right-skewed distribution.

Figure 7 illustrates the CPT utility of MA and buy-and-hold short-selling. Overall, stock investment is attractive for a lot less preference parameter triples. Short-sale according to the 
Figure 7: Utility of Trading MA. This graph illustrates the parameter values for which a CPT investor finds short-selling a stock according to MA attractive. For comparative reasons, part (a) shows the 88 parameter values (indicated by "+") for which trading the stock randomly according to the short-sale benchmark strategy is preferred over no investment. Part (b) compares a short stock investment according to the benchmark strategy, a short stock investment according to MA, and no stock investment. In that case, the benchmark strategy is preferred for 88 parameter combinations (indicated by a "+") while the $\operatorname{MA}(50,200)$ strategy is preferred for 548 parameter combinations (indicated by a "*").

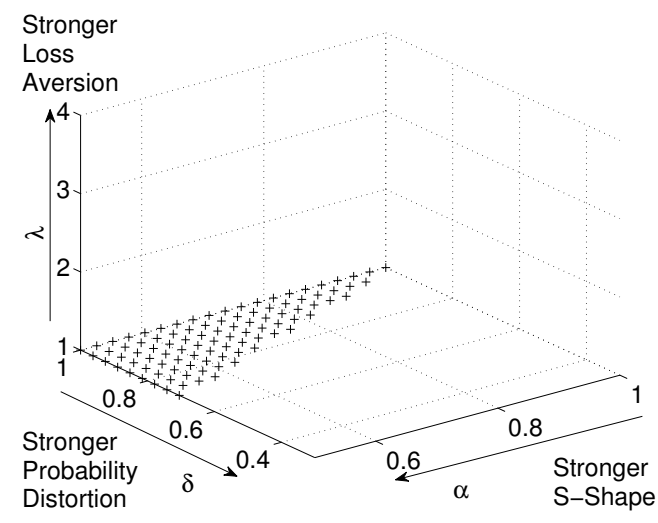

(a) Buy-and-hold vs. no investment

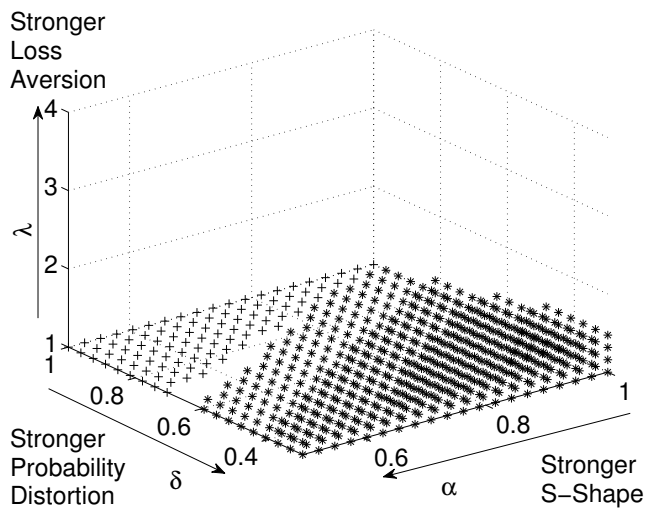

(b) MA vs. buy-and-hold vs. no investment

benchmark strategy (Figure 7a) is preferred over no investment in the case of no loss aversion, mild probability weighting, and pronounced diminishing sensitivity. The left-skewed benchmark distribution implies a small probability of a large loss. But when utility is convex over losses and when the associated probabilities are not overweighted, large losses are not too frightening. Likewise, for these preference parameters, a high probability of small gains is not underweighted. Moreover, small gains with high probability are preferred over large gains with small probability in the sense that large gains are less exciting under diminishing sensitivity. For these reason, CPT investors with low levels of loss aversion and probability weighting as well as with pronounced diminishing sensitivity may prefer left-skewed distributions.

Figure $7 \mathrm{~b}$ illustrates the investment decision when short MA is available. The short MA strategy results in a right-skewed distribution. As discussed, this is preferred for mild loss aversion, mild diminishing sensitivity, and pronounced probability weighting; this is indeed consistent with the position of the "*” marks in Figure $7 \mathrm{~b}$,

Thus our results for the short-sale MA strategy are in line with those we obtained for the case of a long position. No matter whether one employs MA to chase a downward or an upward trend, trading MA skews the distribution of trading proceeds to the right, which is preferred by most CPT investors. The only difference is that, due to the nature of the short-sale, the short-sale MA return distribution is comparatively less right-skewed. This is why, compared to the MA strategy that opens a long position, fewer CPT investors find the short-sale MA strategy attractive. 


\section{Robustness}

In this section, we conduct some additional analysis to evaluate the robustness of our results. In Subsection V.A, we analyze our model for different time horizons. In Subsection V.B, we employ different stock price models with stochastic volatility and jumps. Subsection V.C repeats the analysis for a stock with strictly positive expected return and for different reference points. Subsection V.D studies the case of autocorrelated price dynamics where MA is clearly profitable. Subsection V.E studies MA trading on STOXX Europe 600 stocks since 1990. Subsection V.F employs the MA strategy for short-term MAs of 22 and 37 days. Overall, our observation that trading MA results in a strongly right-skewed return distribution which is appealing to prospect theory investors is qualitatively unaffected by these modifications.

\section{A. Indefinite and Finite Time Horizon}

In the main part of the paper, we assumed that each MA trade is completed. That is, liquidation of the stock exclusively happens at the time of a sell signal, even if the signal is not observed for a long time. In this subsection, we consider the case where the investor may be forced to sell early due to an exogenous liquidity shock. We refer to this time horizon as case 2 while the infinite time horizon considered so far is case 1 . Case 3 then analyzes the situation where the investor has a fixed trading horizon, that is, a final time $T$ at which the stock must be sold at the latest. Finally, in case 4 we compare trading MA with a benchmark buy-and-hold strategy whose holding time is constant and equal to the expected holding time of MA.

Table $\left[\right.$ summarizes these four cases. $\tau_{M A}$ and $\tilde{\tau}_{M A}$ denote the stopping times that refer to the MA and the benchmark strategy, respectively. $\tau_{M A}$ is defined as in Definition 1. $\tilde{\tau}_{M A}$ is identical in distribution to $\tau_{M A}$, but independent of the the stock price dynamics $S_{t}$. The liquidity shock $\tau_{\exp }$ is given by the first arrival time of a Poisson process with parameter $1 /(148 \cdot 1.5)=1 / 222$. Therefore, the liquidity shock is exponentially distributed and occurs on average after 222 days, which is 1.5 times the expected holding time of the MA strategy. We also chose the limited trading horizon in cases 3 to be $T=222$ days. For case 4 we consider a benchmark strategy with a constant holding time which is equal to the average holding time of MA, i.e., $\bar{T}=E\left[\tau_{M A}\right]=148$.

Table I: MA strategy for different Time horizons

\begin{tabular}{c|c|c} 
Time Horizon/Strategy & Double-Crossover MA & Benchmark Buy-and-Hold \\
\hline (1) Infinite (Main Model) & $\tau_{M A}$ & $\tilde{\tau}_{M A}$ \\
(2) Indefinite (Liquidity Shock) & $\tau_{M A} \wedge \tau_{\exp }$ & $\tilde{\tau}_{M A} \wedge \tau_{\exp }$ \\
(3) Finite (fixed trading horizon) & $\tau_{M A} \wedge T$ & $\tilde{\tau}_{M A} \wedge T$ \\
(4) Finite (Constant Benchmark) & $\tau_{M A}$ & $\bar{T}=E\left[\tau_{M A}\right]$
\end{tabular}

Figures 8, 9, and 10 replicate the analysis for the main model (case 1), as shown in Figures 3 and 5 , for cases (2), (3), and (4), respectively. We observe that none of these variations changes 
our observations from the main model qualitatively. As long as the (expected) time horizon for trading is not too short so that a relevant number of MA trades is completed, MA retains its effect of strongly skewing to the right. Further simulations show that, when the (expected) time horizon becomes shorter and shorter, the MA skewness effect diminishes more and more.

\section{B. Stochastic Volatility and Jumps}

In this subsection, we relax the assumption of geometric Brownian motion and study MA when prices evolve according to the popular stochastic volatility model of Heston (1993) and the jumpdiffusion model of Merton (1976). According to the Heston model, the stock price dynamics are given by

$$
\begin{aligned}
& d S_{t}=\mu S_{t} d t+\sqrt{V_{t}} S_{t} d W_{t}^{S}, S_{0}>0 \\
& d V_{t}=\kappa\left(\theta-V_{t}\right) d t+\sigma_{v o l} \sqrt{V_{t}} W_{t}^{V}, V_{0}>0 .
\end{aligned}
$$

Here, $\mu$ denotes the drift of the stock price $S_{t}$ which is driven by a Brownian motion $W^{S}$ whose volatility is stochastic. The volatility process $V$ is driven by a Brownian motion $W^{V}$ with a volatility of volatility $\sigma_{v o l}$, mean reversion level $\theta$, and speed of reversion $\kappa$. The two Brownian motions are correlated through a parameter $\rho$. Figure 11 illustrates our results for the Heston model with $\mu=0$, $\sigma_{v o l}=0.5, \rho=-0.7, \theta=0.3, \kappa=3$, and $V_{0}=0.5$.

In the jump-diffusion model of Merton (1976), the stock price dynamics and the solution to the stochastic differential equation are as follows:

$$
\begin{aligned}
d S(t) & =(\mu-\gamma \kappa) S(t-) d t+\sigma_{S} S(t-) d W_{t}^{S}+(Y(t)-1) d N(t), \quad S_{0}>0 \\
S(t) & =S_{0} \exp \left(\left(\mu-\gamma \kappa-\frac{\sigma_{S}^{2}}{2}\right) t+\sigma_{S} W(t)\right) \Pi_{i=1}^{N(t)} Y\left(t_{i}\right)
\end{aligned}
$$

Here, $\mu$ denotes the drift of the stock price $S_{t}$ which is driven by the Brownian motion $W^{S}$ with volatility $\sigma_{S} . N(t)$ is a Poisson process, where $N_{t}$ denotes the number of jumps until time $t$ which are computed with jump intensity of $\gamma$, that is the mean number of arrivals per year. The jump size $Y_{i}$ of jump $i$ follows a lognormal distribution with mean zero and a volatility of $\sigma_{\text {jump }}$. Figure 12 presents the results for a Merton model with $\mu=0, \sigma=0.3, \sigma_{\text {jump }}=0.15$ and $\gamma=1 . \kappa=\mathbb{E}[Y-1]$ is the expected relative change in the stock upon arrival of a price jump. 
Figure 8: MA trading in the presence of liquidity shocks. Part (a) of this figure illustrates the return distributions of $M A$ (solid line) and the benchmark strategy (dashed line) when investing is subject to a liquidity shock that, on average, occurs after 222 days (case 2 in Table I). The skewness of the MA (benchmark) return distribution is 2.92 (1.02) and the expected holding time of both strategies is 94 days. Part (b) shows the 730 CPT parameter values (indicated by "+") for which the benchmark strategy is preferred over no investment. Part (c) compares stock investment according to the benchmark strategy, stock investment according to $M A$, and no stock investment. In that case, the benchmark strategy is preferred for 24 parameter combinations (indicated by a " + ") while the $M A(50,200)$ strategy is preferred for 1869 parameter combinations (indicated by a "*").

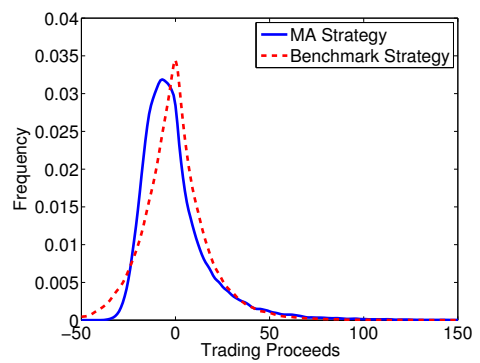

(a) Density

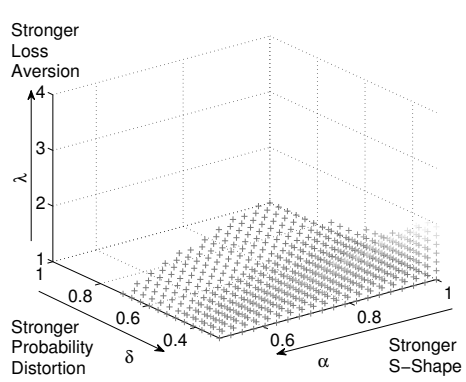

(b) Buy-and-hold vs. no investment

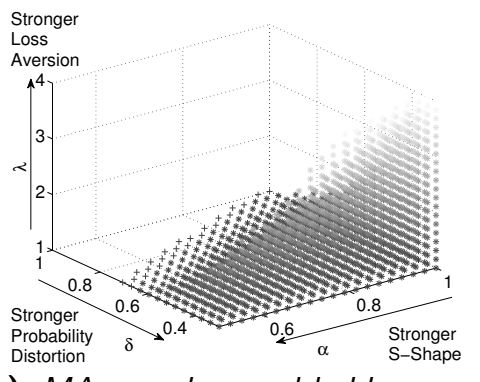

(c) MA vs. buy-and-hold vs. no

investment

Figure 9: MA trading with a fixed trading horizon. Part (a) of this figure illustrates the return distributions of MA (solid line) and the benchmark strategy (dashed line) when the investor has a limited trading horizon 222 days (case 3 in Table (1). The skewness of the MA (benchmark) return distribution is 2.01 (0.88) and the expected holding time of both strategies is 123 days. Part (b) shows the 733 CPT parameter values (indicated by "+") for which the benchmark strategy is preferred over no investment. Part (c) compares stock investment according to the benchmark strategy, stock investment according to $M A$, and no stock investment. In that case, the benchmark strategy is preferred for 44 parameter combinations (indicated by a " + ") while the MA(50,200) strategy is preferred for 1425 parameter combinations (indicated by $a$ “*”).

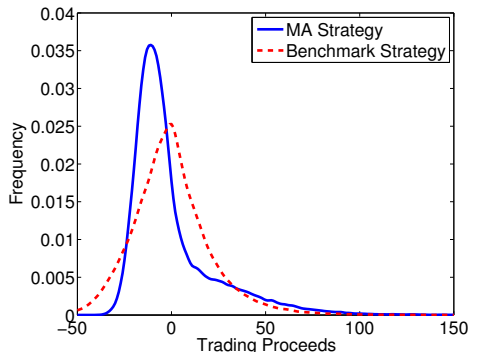

(a) Density

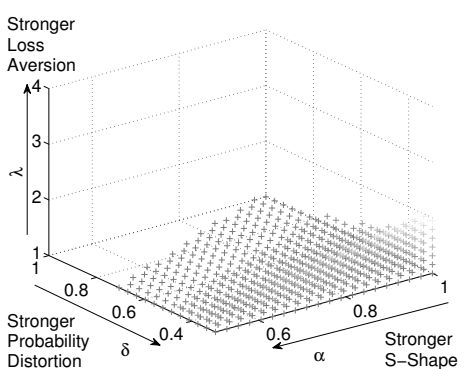

(b) Buy-and-hold vs. no investment $\begin{aligned} & \text { (c) } M A \text { vs } \\ & \text { investment }\end{aligned}$

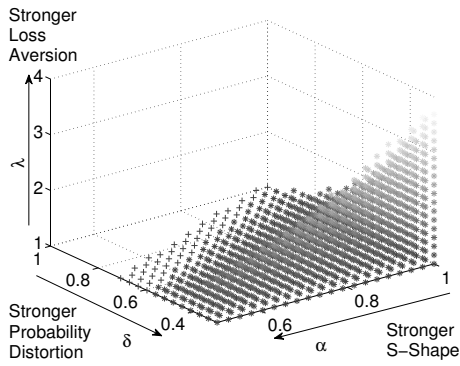


Figure 10: MA trading for a constant Benchmark. Part (a) of this figure illustrates the return distributions of MA (solid line) and a buy-and-hold benchmark strategy (dashed line) with a constant holding time equal to 148 days (case 4 in Table 1). The skewness of the MA (benchmark) return distribution is 4.61 (0.7) and the expected holding time of both strategies is 148 days. Part (b) shows the 683 CPT parameter values (indicated by "+") for which the benchmark strategy is preferred over no investment. Part (c) compares stock investment according to the benchmark strategy, stock investment according to MA, and no stock investment. In that case, the benchmark strategy is preferred for 25 parameter combinations (indicated by a " + ") while the MA(50,200) strategy is preferred for 2486 parameter combinations (indicated by a "*").

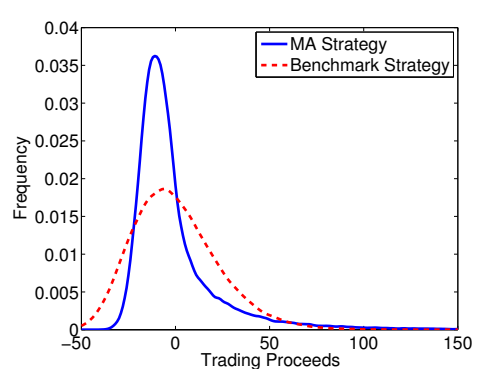

(a) Density

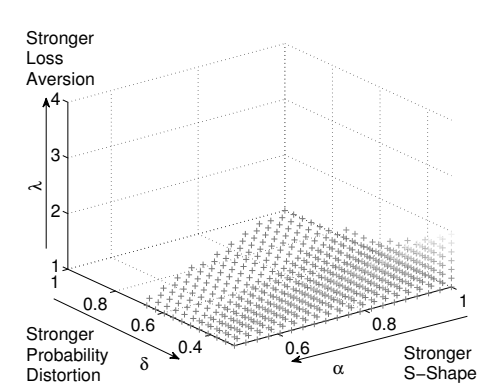

(b) Buy-and-hold vs. no investment

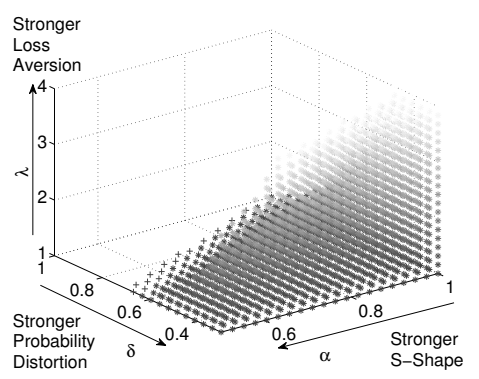

(c) MA vs. buy-and-hold vs. no investment

Figure 11: MA trading in the Heston Model. Part (a) of this figure shows the return distributions of MA (solid line) and the benchmark strategy (dashed line) when the stock price follows the dynamics of a Heston model. The skewness of the MA (benchmark) return distribution is 10.65 (2.17) and the expected holding time of both strategies is 135 days. Part (b) shows the 1519 CPT parameter values (indicated by "+") for which the benchmark strategy is preferred over no investment. Part (c) compares stock investment according to the benchmark strategy, stock investment according to $M A$, and no stock investment. In that case, the benchmark strategy is preferred for 15 parameter combination while $\operatorname{MA}(50,200)$ is preferred for 3008 parameter combinations (indicated by a "*").

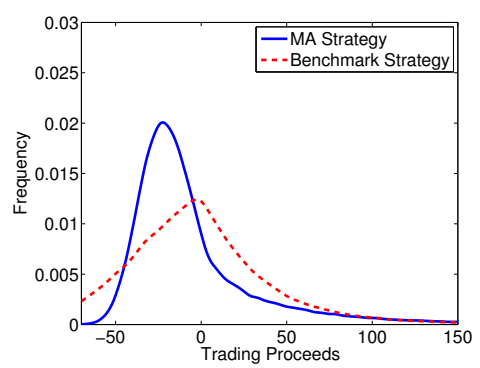

(a) Density

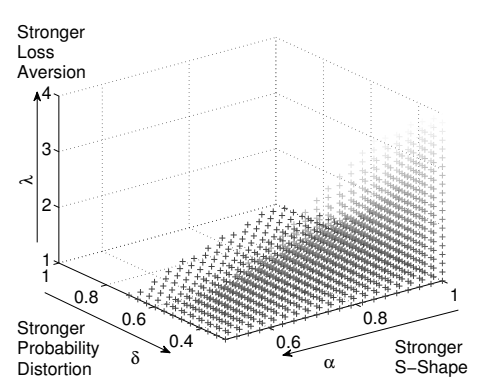

(b) Buy-and-hold vs. no investment

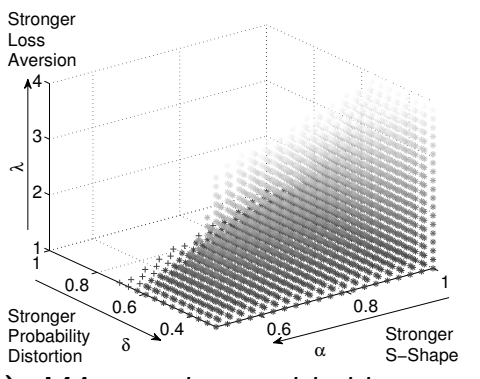

(c) MA vs. buy-and-hold vs. no 
Figure 12: MA trading in the Merton jump-diffusion Model. Part (a) of this figure shows the return distributions of MA (solid line) and the benchmark strategy (dashed line) when the stock price follows the dynamics of a Heston model. The skewness of the MA (benchmark) return distribution is 5.69 (1.64) and the expected holding time of both strategies is 149 days. Part (b) shows the 1194 CPT parameter values (indicated by "+") for which the benchmark strategy is preferred over no investment. Part (c) compares stock investment according to the benchmark strategy, stock investment according to $M A$, and no stock investment. In that case, the benchmark strategy is preferred for 60 parameter combinations while $M A$ is preferred for 2680 parameter combinations (indicated by a "*").

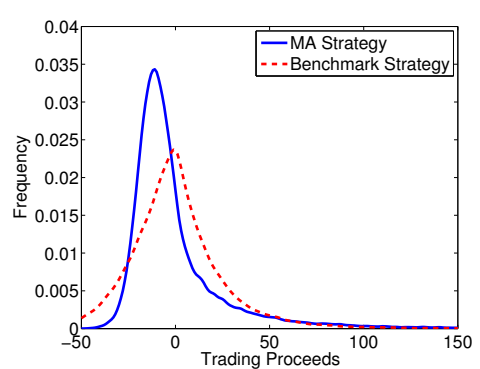

(a) Density

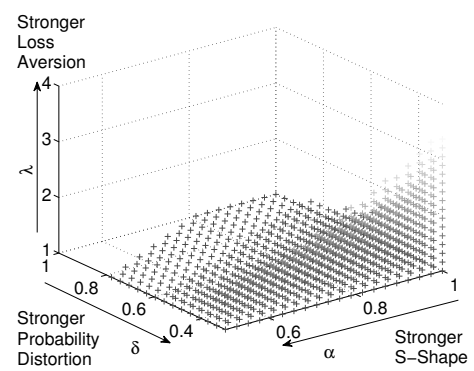

(b) Buy-and-hold vs. no investmen

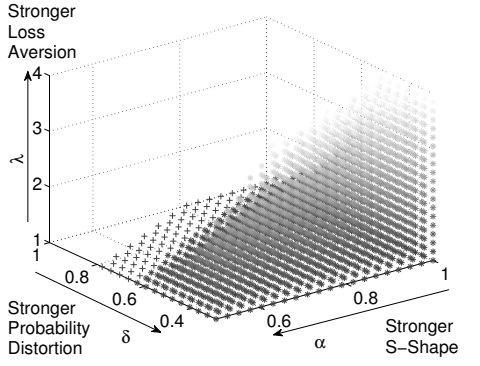

(c) MA vs. buy-and-hold vs. no 


\section{Excess Return}

In this section, we study the more realistic situation when the stock offers excess return. In this case, Doob's optional sampling theorem does not apply and various trading strategies offer different expected returns. We thus present the CPT analysis for a reference point of zero (Figure 13) and also for the case where the reference point equals the expected return of the MA strategy (Figure 14).

As the stock now offers an excess return, both the MA and the benchmark strategy are (unconditionally) profitable. In the studied scenario, the MA strategy (the benchmark strategy) yields an expected return of $E\left[\tau_{M A},\right]=2.09 \%\left(E\left[\tau_{\tilde{M} A}\right]=1.92 \%\right)$ over an expected holding period of 155 days. The observation that trading MA results in a strongly skewed return distribution remains. A comparison of Figures 13 and 14 evidences that differences in reference points (in magnitudes that are economically meaningful) are not crucial to our result that the CPT investor has a pronounced preference for the right-skewed MA distribution.

Figure 13: MA trading in the GBM model with trend and a reference point of zero. Part (a) of this figure shows the return distributions of MA (solid line) and the benchmark strategy (dashed line) when the stock price follows the dynamics of a GBM model with a positive trend of $\mu=0.03$. The skewness of the MA (benchmark) return distribution is $7.88(1,62)$ and the expected holding time of both strategies is 155 days. Part (b) shows the 1592 CPT parameter values (indicated by " + ") for which the benchmark strategy is preferred over no investment. Part (c) compares stock investment according to the benchmark strategy, stock investment according to MA, and no stock investment. In that case, the benchmark strategy is preferred for 120 parameter combinations while MA is preferred for 3174 parameter combinations (indicated by a “*”).

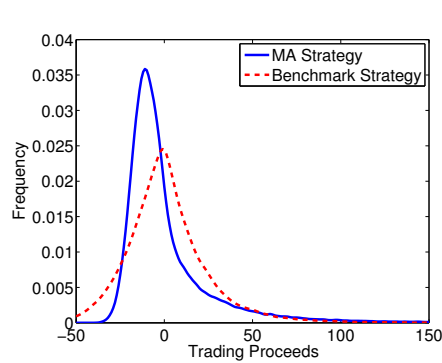

(a) Density

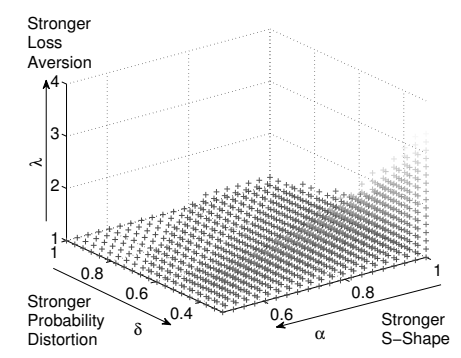

(b) Buy-and-hold vs. no investment

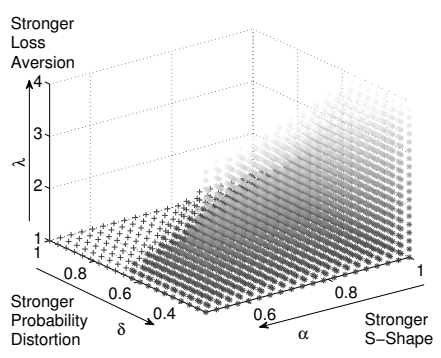

(c) MA vs. buy-and-hold vs. no investment 
Figure 14: MA trading in the GBM model with trend when the reference point equals expected return. Part (a) of this figure shows the return distributions of $M A$ (solid line) and the benchmark strategy (dashed line) when the stock price follows the dynamics of a GBM model with a positive trend of $\mu=0.03$; cf. Figure $13 a$ Part (b) shows the 719 CPT parameter values (indicated by " + ") for which the benchmark strategy is preferred over no investment. Part (c) compares stock investment according to the benchmark strategy, stock investment according to MA, and no stock investment. In that case, the benchmark strategy is preferred for zero parameter combinations while MA is preferred for 2355 parameter combinations (indicated by a "*").

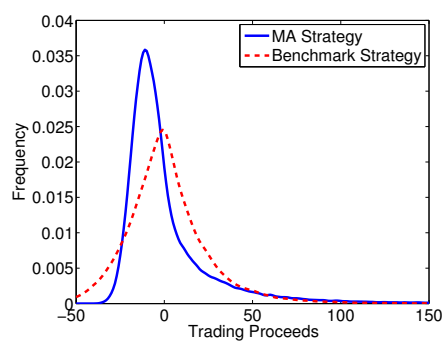

(a) Density

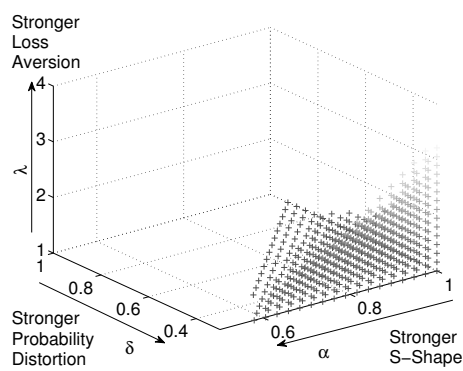

(b) Buy-and-hold vs. no investmen

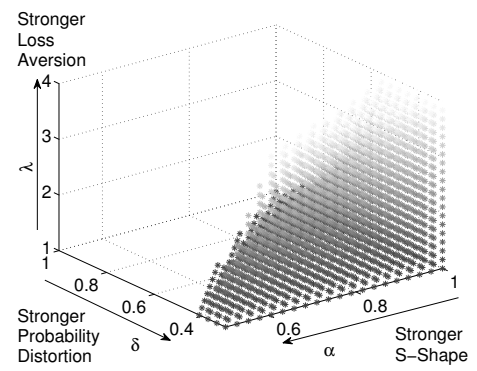

(c) MA vs. buy-and-hold vs. no

investment 


\section{Autocorrelated Returns}

In this section, we assume stock price dynamics that imply that prices do move in trends over a time period of several months such that the MA strategy indeed is indeed profitable. Specifically, we employ a Markov-switching model as an extension of the geometric Brownian motion model: The stock $S$ has the following dynamics:

$$
d S_{t}=S(t)[\mu \cdot \eta(t) d t+\sigma d W(t)]
$$

Here, the stock drift $\mu$ is a function of the regime $\eta$. $\eta$ can take the values +1 and -1 , specifying a positive or negative regime. The switching probabilities are equal, that is, a switch from positive to negative regime and from negative to positive regime are equal. Note that the process is a submartingale for the parametrzation in Figure 15. The switching probabilities are $0.5 \%$ per day, resulting in a mean of 1.3 switches per year.

Figure 15: MA trading in a model of autocorrelated returns. Part (a) of this figure shows the return distributions of MA (solid line) and the benchmark strategy (dashed line) when the stock price evolves according to an autocorrelated Markov switching model. The return of the MA (benchmark) strategy is 0.53 (0.066). The skewness of the MA (benchmark) return distribution is 5.42 (1.45) and the expected holding time of both strategies is 148 days. Part (b) shows the 977 CPT parameter values (indicated by "+") for which the benchmark strategy is preferred over no investment. Part (c) compares stock investment according to the benchmark strategy, stock investment according to MA, and no stock investment. In that case, the benchmark strategy is preferred for 19 parameter combinations while MA is preferred for 2726 parameter combinations (indicated by a "*”).

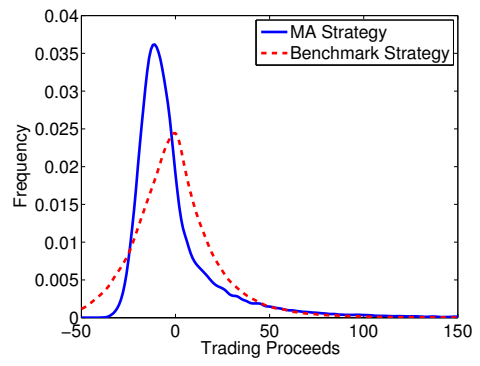

(a) Density

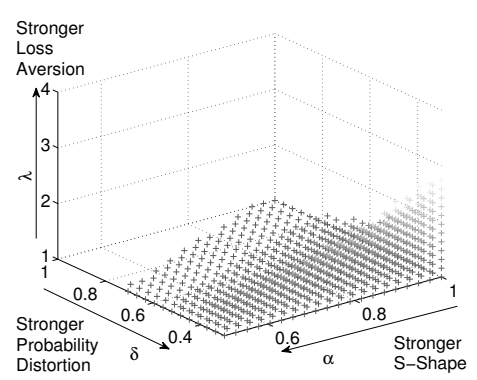

(b) Buy-and-hold vs. no investment

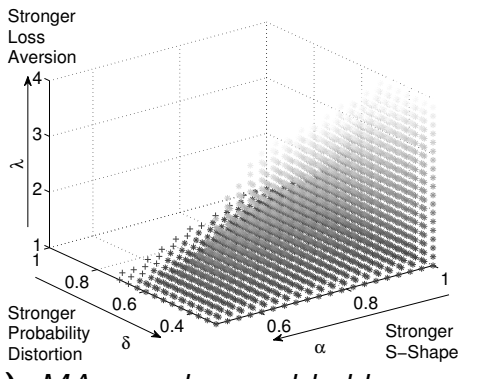

(c) MA vs. buy-and-hold vs. no

\section{E. Trading MA on Real Data}

In this section, we compute and analyze the MA return distribution based on real stock price data. We consider all stocks that were part of the STOXX Europe 600 at January 1, 2000 and monitor these stocks until June 30, 2013 or until de-listed. The MA return distribution is obtained as the empirical distribution of all possible MA trades of all 600 stocks within this sample period. For each stock we observe on average 7.1 buy signals that are followed by a sell signal in the sample period, which implies a total number of 4283 trades. The average holding time of a trade equals 132 days. The return distribution for the random buy-and-hold benchmark strategy is constructed by sampling the same number of 4283 trades as follows: pick a stock at random, draw a buy time 
uniformly over the sample period and draw a holding time from the same distribution as the MA strategies' holding time.

Figure 16: MA trading on STOXX Europe 600 stocks. Part (a) of this figure shows the return distributions of MA (solid line) and the benchmark strategy (dashed line) obtained from trading MA all stocks that were part of the STOXX Europe 600 at 1 January, 2000 until 30 June, 2013 or until de-listed. The mean return for the MA (benchmark) strategy is 4.35 (1.43) for the benchmark strategy for an average holding time of 133 days. The skewness of the MA (benchmark) return distribution is 6.82 (4.89). Part (b) shows the 1642 CPT parameter values (indicated by " + ") for which the benchmark strategy is preferred over no investment. Part (c) compares stock investment according to the benchmark strategy, stock investment according to $M A$, and no stock investment. In that case, the benchmark strategy is preferred for 37 parameter combinations while MA is preferred for 3636 parameter combinations (indicated by a “*”).

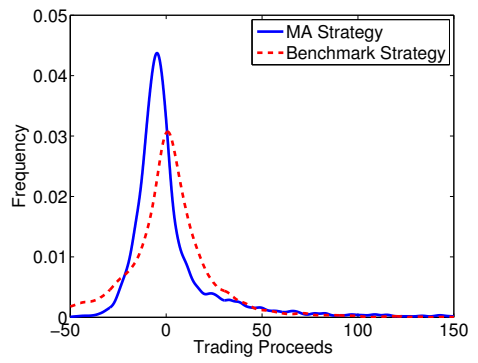

(a) Density

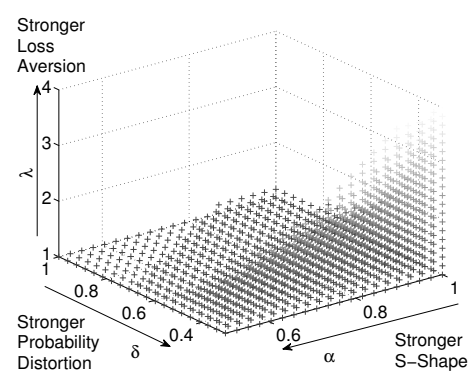

(b) Buy-and-hold vs. no investment

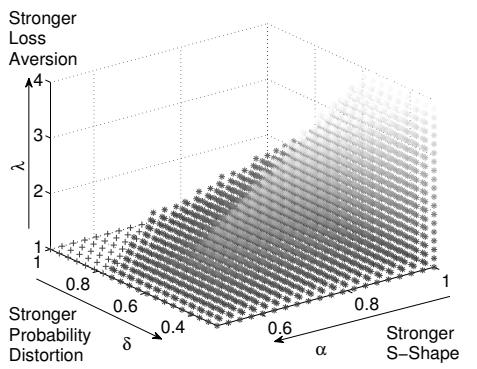

(c) MA vs. buy-and-hold vs. no

investment

\section{F. Timing of the MA Strategy}

In this section, we repeat our analysis for a MA strategy that is based on shorter short-term MA lines of length 22 and 37, respectively, while the the long-term MA line remains at 200 days. Furthermore, we repeat our analysis for a MA strategy that has a short long-term MA line of length 100, while the short-term MA remains at 50. The results are shown in Figures 17, 18, and [20. Shorter short-term MAs actually amplify the skewness effect of MA we observe, as stocks that start losing in value are sold even more quickly. The general observation is that the larger the difference in length between the long-term and short-term MA, i.e., the more flexible the short-term MA and the more sluggish the long-term MA, the more does MA skew to the right. This we illustrate also in Figure 19. 
Figure 17: MA trading with a 37-day short-term MA. Part (a) of this figure shows the return distributions of MA(37,200) (solid line) and the benchmark strategy (dashed line). The skewness of the MA (benchmark) return distribution is 5.13 (1.27) and the expected holding time of both strategies is 132 days. Part (b) shows the 895 CPT parameter values (indicated by " + ") for which the benchmark strategy is preferred over no investment. Part (c) compares stock investment according to the benchmark strategy, stock investment according to MA, and no stock investment. In that case, the benchmark strategy is preferred for 26 parameter combinations while MA is preferred for 2586 parameter combinations (indicated by a “*”).

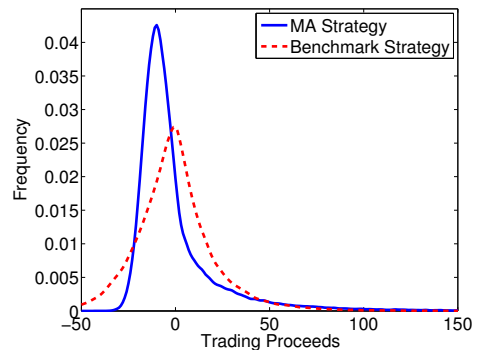

(a) Density

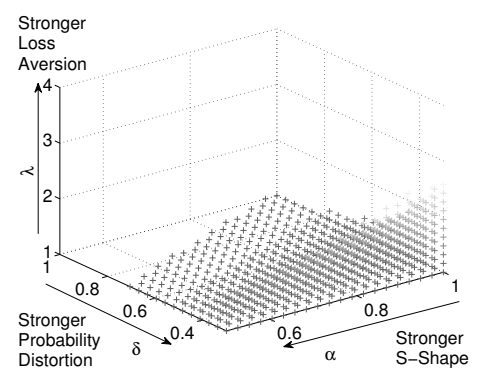

(b) Buy-and-hold vs. no investment

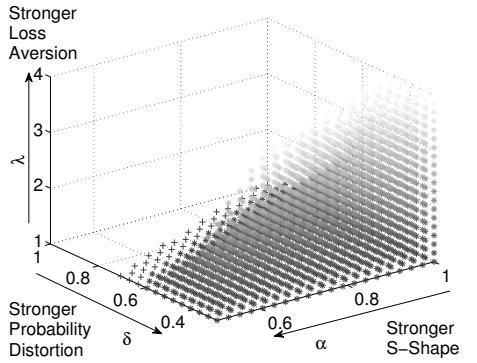

(c) MA vs. buy-and-hold vs. no investment

Figure 18: MA trading with a 22-day short-term MA. Part (a) of this figure shows the return distributions of MA(22,200) (solid line) and the benchmark strategy (dashed line). The skewness of the MA (benchmark) return distribution is 5.68 (1.56) and the expected holding time of both strategies is 109 days. Part (b) shows the 863 CPT parameter values (indicated by " + ") for which the benchmark strategy is preferred over no investment. Part (c) compares stock investment according to the benchmark strategy, stock investment according to MA, and no stock investment. In that case, the benchmark strategy is preferred for 29 parameter combinations while MA is preferred for 2769 parameter combinations (indicated by $a^{\text { “*”). }}$.

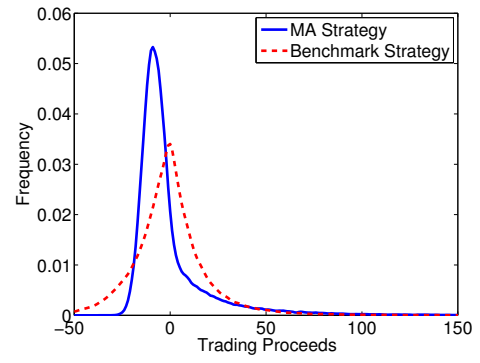

(a) Density

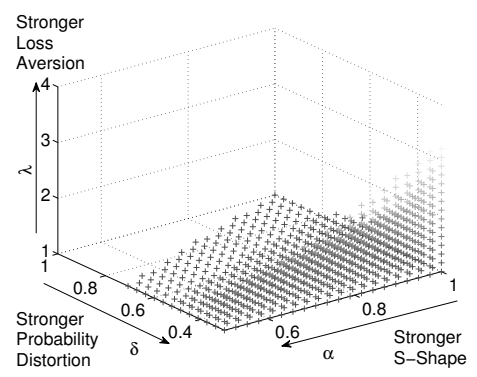

(b) Buy-and-hold vs. no investment

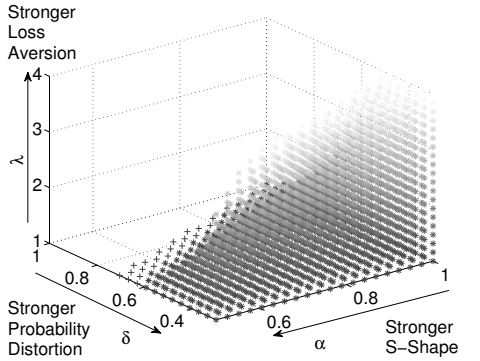

(c) MA vs. buy-and-hold vs. no

investment

Figure 19: Comparison of distributions from different MA strategies. This graph shows the densities implied by the $\operatorname{MA}(50,200)$ (solid), the $\operatorname{MA}(37,200)$ (dashed), and the MA(22,200) (dotted) strategies.

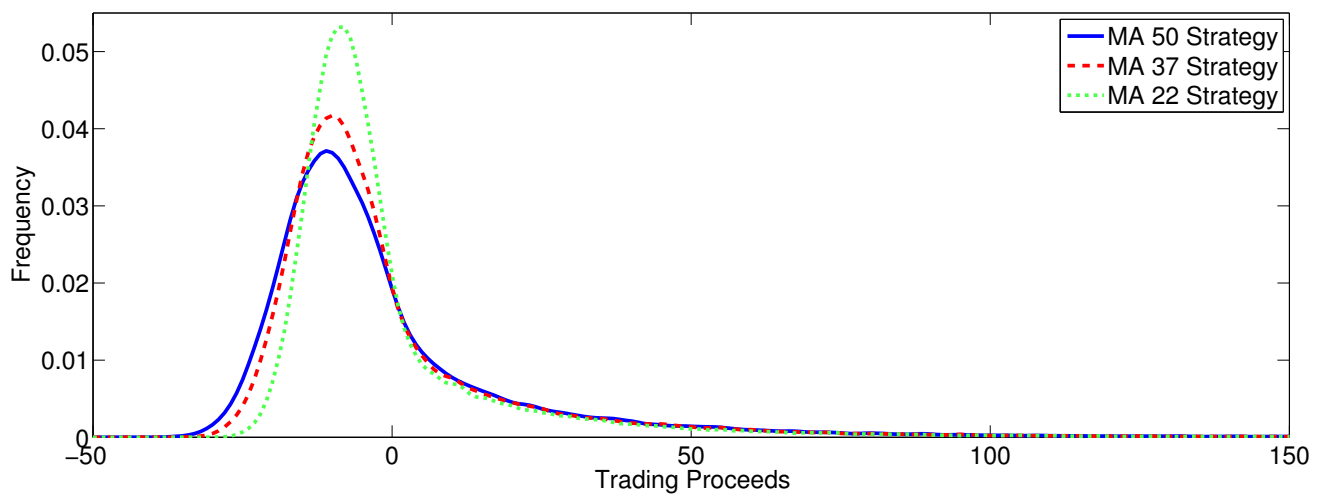


Figure 20: MA trading with a 100-day long-term MA. Part (a) of this figure shows the return distributions of MA(50,100) (solid line) and the benchmark strategy (dashed line). The skewness of the MA (benchmark) return distribution is 4.08 (0.90) and the expected holding time of both strategies is 88 days. Part (b) shows the 679 CPT parameter values (indicated by " + ") for which the benchmark strategy is preferred over no investment. Part (c) compares stock investment according to the benchmark strategy, stock investment according to MA, and no stock investment. In that case, the benchmark strategy is preferred for 27 parameter combinations while MA is preferred for 2138 parameter combinations (indicated by a “*”).

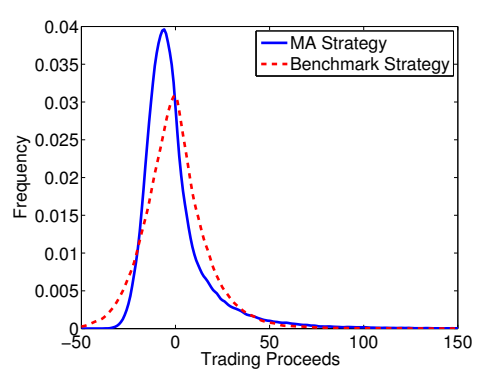

(a) Density

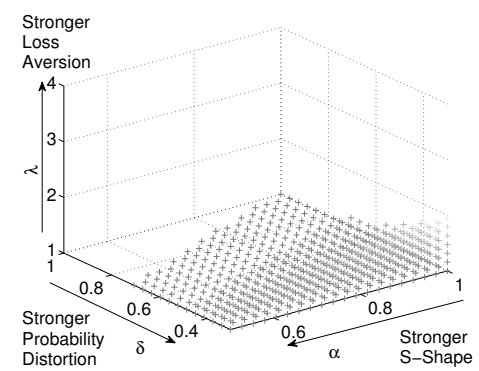

(b) Buy-and-hold vs. no investmen

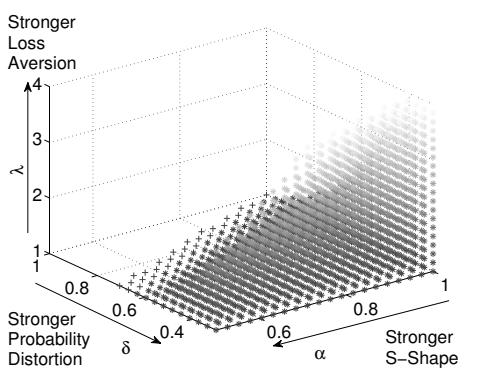

(c) MA vs. buy-and-hold vs. no 


\section{Conclusion}

We have analyzed one of the most popular, trend-chasing technical trading strategies - the double-crossover moving average strategy - in a behavioral setting. Even when prices do not move in trends, when trading MA is not profitable, and when rational expected utility maximizers thus not trade MA, the popularity of MA can be reconciled with cumulative prospect theory preferences. While academics are generally skeptical towards technical analysis, we believe that this paper is the first to show that technical analysis can be exclusively reconciled from less than fully rational preferences.

Our result is a consequence of the observation that trading MA skews the distribution of trading proceeds to the right. This is attractive to many prospect theory investors who have strong preferences for right-skewed payoff distributions.

While we obtain this result explicitly for the MA strategy, we believe that the same rationale also applies to other trading strategies that chase momentum. That is, it lies in the nature of trend-chasing that one sells quickly upon a downward trend and holds on to the position in case of an upward trend. Chasing momentum thus makes small losses likely but also generates a small possibility of large gains. In other words, chasing trends results in right-skewed payoff distributions. The prevalence of momentum chasing may thus not only be explained with prices actually moving in trends - or a belief therein - but also by investors having strong preferences for positive skewness.

We conducted our analysis for the most prominent model of less than fully rational investor preferences: prospect theory. For most parametrizations that are observed in empirical settings, prospect theory implies skewness preference. However, other behavioral theories that imply skewness preference may just as well explain the attractiveness of trend-chasing. For example, the

salience model of choice under risk by Bordalo, Gennaioli, and Shleifer $(2012,2013)$ also implies a taste for positive skewness and is thus a promising candidate to imply a preference for trend-chasing.

\section{REFERENCES}

Barberis, N., 2012, A model of casino gambling, Management Science 58, 35-51.

Barberis, N., 2013, The psychology of tail events: Progress and challenges, American Economic Review Papers and Proceedings 103, 611-616.

Barberis, N., and M. Huang, 2008, Stocks as lotteries: The implications of probability weighting for security prices, American Economic Review 98, 2066-2100.

Barberis, N., and W. Xiong, 2012, Realization utility, Journal of Financial Economics 104, 251-271. 
Barberis, N., and Wei Xiong, 2009, What drives the disposition effect? an analysis of a longstanding preference-based explanation, Journal of Finance 64, 751-784.

Barberis, Nicholas, Andrei Shleifer, and Robert Vishny, 1998, A model of investor sentiment, Journal of Financial Economics 49.

Bordalo, P., N. Gennaioli, and A. Shleifer, 2012, Salience theory of choice under risk, Quarterly Journal of Economics 127, 1243-1285.

Bordalo, P., N. Gennaioli, and A. Shleifer, 2013, Salience and asset prices, American Economic Reviw: Papers $\&$ Proceedings 103, 623-628.

De Long, J. Bradford, Andrei Shleifer, Lawrence H. Summers, and Robert Vishny, 1990, Noise trader risk in financial markets, Journal of Political Economy 98, 703-738.

Ebert, S., and P. Strack, 2012, Until the bitter end: On prospect theory in the dynamic context, Working Paper, University of Bonn.

Fama, Eugene F., 1970, Efficient capital markets: A review of theory and empirical work, Journal of Finance 25, 383-417.

Green, T.C., and B.-H. Hwang, 2012, Initial public offerings as lotteries: Skewness preference and first-day returns, Management Science 58, 432-444.

Grinblatt, Mark, and Bing Han, 2005, Prospect theory, mental accounting, and momentum, Journal of Financial Economics 78, 311-339.

Henderson, V., 2012, Prospect theory, liquidation and the disposition effect, Management Science $58,445-460$.

Heston, Steven L., 1993, A closed-form solution for options with stochastic volatility with applications to bond and currency options, Review of Financial Studies 6, 327-343.

Jegadeesh, Narasimhan, and Sheridan Titman, 1993, Returns to buying winners and selling losers: Implications for stock market efficiency, Journal of Finance 48, 65-91.

Jegadeesh, Narasimhan, and Sheridan Titman, 2001, Profitability of momentum strategies: An evaluation of alternative explanations, Journal of Finance 56, 699-720. 
Jensen, Michael C., 1978, Some anomalous evidence regarding market efficiency, Journal of Financial Economics 6, 95-101.

Kahneman, Daniel, and Amos Tversky, 1979, Prospect Theory: An Analysis of Decision under Risk, Econometrica 47, 263-292.

Menkhoff, Lukas, 2010, The use of technical analysis by fund managers: International evidence, Journal of Banking and Finance 34, 2573-2586.

Menkhoff, Lukas, Lucio Sarno, Maik Schmeling, and Andreas Schrimpf, 2012, Currency momentum strategies, Journal of Financial Economics 106, 660-684.

Menkhoff, Lukas, and Mark P. Taylor, 2007, The obstinate passion of foreign exchange professionals: Technical analysis, Journal of Economic Literature 45, 936-972.

Merton, R. C., 1976, Option pricing when underlying stock returns are discontinuous, Journal of Financial Economics 3, 125-144.

Park, Cheol-Ho, and Scott H. Irwin, 2007, What do we know about the profitabiltiy of technical analysis, Journal of Economic Surveys 21, 786-826.

Rieger, M. O., and M. Wang, 2006, Prospect theory and the st. petersburg paradox., Economic Theory 28, 665-679.

Schneider, C., and O. Spalt, 2012, Conglomerate investment, skewness, and the ceo long shot bias, Working Paper.

Shefrin, H., and M. Statman, 1985, The disposition to sell winners too early and ride losers too long: Theory and evidence, Journal of Finance 40, 777-790.

Shleifer, Andrei, and Robert Vishny, 1997, The limits of arbitrage, Journal of Finance 52, 35-55.

Tversky, A., and D. Kahneman, 1992, Advances in prospect theory: Cumulative representation of uncertainty, Journal of Risk and Uncertainty 5, 297-323.

Zhu, Y., and G. Zhou, 2009, Technical analysis: An asset allocation perspective on the use of moving averages, Journal of Financial Economics 92, 519-544. 